\title{
Cytoplasmic incompatibility between Old and New World populations of a tramp ant
}

\author{
Çiğdem Ün, ${ }^{1,+(1)}$ Eva Schultner, ${ }^{1,2,+}$ (D) Alejandro Manzano-Marín, ${ }^{3}$ (D) Laura V. Flórez, ${ }^{4}$ (D) Bernhard Seifert, ${ }^{5}$ \\ Jürgen Heinze, ${ }^{1}$ (D) and Jan Oettler ${ }^{1,6}$ (D) \\ 'Zoology/Evolutionary Biology, University of Regensburg, Regensburg 93053, Germany \\ ${ }^{2}$ E-mail: eva.schultner@gmail.com \\ ${ }^{3}$ Centre for Microbiology and Environmental Systems Science, University of Vienna, Vienna 1090, Austria \\ ${ }^{4}$ Institute of Organismic and Molecular Evolution, Evolutionary Ecology Department, Johannes Gutenberg University \\ Mainz, Mainz 55128, Germany \\ ${ }^{5}$ Senckenberg Museum of Natural History Görlitz, Görlitz 02826, Germany \\ ${ }^{6}$ E-mail: joettler@gmail.com
}

Received June 21, 2020

Accepted April 16, 2021

\begin{abstract}
Reproductive manipulation by endosymbiotic Wolbachia can cause unequal inheritance, allowing the manipulator to spread and potentially impacting evolutionary dynamics in infected hosts. Tramp and invasive species are excellent models to study the dynamics of host-Wolbachia associations because introduced populations often diverge in their microbiomes after colonizing new habitats, resulting in infection polymorphisms between native and introduced populations. Ants are the most abundant group of insects on earth, and numerous ant species are classified as highly invasive. However, little is known about the role of Wolbachia in these ecologically dominant insects. Here, we provide the first description of reproductive manipulation by Wolbachia in an ant. We show that Old and New World populations of the cosmotropic tramp ant Cardiocondyla obscurior harbor distinct Wolbachia strains, and that only the Old World strain manipulates host reproduction by causing cytoplasmic incompatibility (Cl) in hybrid crosses. By uncovering a symbiont-induced mechanism of reproductive isolation in a social insect, our study provides a novel perspective on the biology of tramp ants and introduces a new system for studying the evolutionary consequences of $\mathrm{Cl}$.
\end{abstract}

KEY WORDS: antibiotics, endosymbiont, reproductive isolation, social insects, Wolbachia.

Symbiotic associations between organisms are widespread in nature. The bacterium Wolbachia infects around $40 \%$ of the world's arthropod species (Zug and Hammerstein 2012) and is best known for its role as a maternally inherited endosymbiont that manipulates host reproduction to ensure its own transmission. The most common of these reproductive strategies is cytoplasmic incompatibility (CI), which occurs because Wolbachia can modify the sperm of infected males. In eggs from infected females, a rescue factor reverses this modification, allowing embryogenesis to proceed normally (Shropshire et al. 2020). When the rescue factor is missing because females are not infected or infected with a different Wolbachia strain (Yen and Barr 1971; Werren

${ }^{\dagger}$ These authors contributed equally to this work. et al. 2008), embryogenesis is interrupted, typically resulting in embryonic death. Within a population, infected females thus have a reproductive advantage over uninfected females, which allows the symbiont to spread. Reproductive manipulation by CI-inducing Wolbachia may also have important consequences for the infected host species. This is because CI can affect gene flow between populations that differ in their infection status, potentially facilitating genetic differentiation and in some cases even contributing to speciation (Engelstädter and Telschow 2009; Brucker and Bordenstein 2012). In spite of the potential impact of CI on host evolutionary dynamics, apart from the classic models Culex, Drosophila, and Nasonia, few insect systems are available that offer all the ingredients needed for study of this 
phenomenon, including detailed knowledge of life history, robust population genomic and phenotypic data, natural diversity in Wolbachia infection, and easy manipulation in the lab.

Tramp and invasive species are emerging models to study the dynamics of insect-endosymbiont associations and their potential effects on host evolution. When introduced to new habitatstypically as a result of human activities-founding populations go through bottlenecks, which reduce diversity within, while at the same time increasing differentiation between, native and introduced populations (Tsutsui et al. 2000). This reduction in diversity applies both to the genetic makeup of the host and to its microbiome (Morrow et al. 2017). Coupled with exposure of the host to new microbial communities in the novel habitats, native and introduced populations can quickly diverge in their microbiomes (Goryacheva et al. 2016; Zepeda-Paulo et al. 2018; Parker et al. 2020). In the case of Wolbachia, horizontal transmission events are common (Schuler et al. 2013; Ahmed et al. 2016), and likely contribute to rapid turnover of Wolbachia strains in introduced populations. Persistence of newly acquired strains may then be reinforced by selective advantages in the novel environment (Versace et al. 2014), or because infection has positive effects on fecundity (Fry et al. 2004). If native and introduced populations continue to evolve in strict geographical isolation, the mere absence of gene flow may eventually result in genetic differentiation and potentially speciation, irrespective of Wolbachia infection. However, tramp and invasive species are often repeatedly moved by human traffic, resulting in regular secondary contact between native and introduced populations, or between distinct introduced populations. When populations that have diverged in Wolbachia infection meet in such secondary contact zones, the dynamics of infection can be exposed (Lis et al. 2015).

Ants represent some of the worst invasive species in the world, and Wolbachia infection is widespread across the ant phylogeny (Russell 2012). Still, very little is known about the role of the symbiont in these ecologically dominant insects. The tramp ant Cardiocondyla obscurior has been spread by human traffic from its putative origin in Southeast Asia to fruit tree plantations, city parks, and other disturbed habitats in the tropics and subtropics around the world (Heinze et al. 2006). A study using ants collected from populations in Brazil and Japan showed that mating between queens and males from the two populations readily occurs in the laboratory. A phenotype indicative of reproductive isolation was found in hybrid crosses between Brazilian queens and Japanese males, with queens exhibiting shorter lifespans and colonies producing fewer offspring than in reciprocal crosses between Japanese queens and Brazilian males and in intrapopulation crosses between Brazilian queens and Brazilian males (Schrempf et al. 2015). At the time, this was attributed to incompatibilities between fast evolving sexual traits of the host such as seminal fluid proteins, a result seemingly linked to an upregulation of genes involved with immune response in outbred Brazilian queens (Schrempf et al. 2015).

Here, we show that the negative effect on ant fitness is in fact caused by incompatibility between Wolbachia strains harbored by the two populations. By reducing Wolbachia levels in Japanese males using antibiotic treatment, we demonstrate that the fitness of Brazilian outbred queens can be rescued. This effect occurred irrespective of whether males were infected with the second main bacterial endosymbiont, Candidatus Westeberhardia cardiocondylae, which exhibits characteristics of an obligate symbiont such as vertical maternal transmission and a reduced genome, but has been lost in one population in Japan (Klein et al. 2015). Genomic analyses of the Wolbachia strains isolated from the two populations reveal that both strains belong to Wolbachia supergroup A, which typically contains strains capable of reproductive manipulation. Surprisingly, CI is only induced by the Wolbachia strain isolated from the Japanese population. The ability to induce CI is reflected in higher Wolbachia titers in ants from Japanese colonies and may be linked to functional differences in CI-associated loci. The putative origin of $C$. obscurior in Southeast Asia, together with sequence identity between Wolbachia strains isolated from populations in Japan and Taiwan, suggests that an association with the Japanese strain is ancestral in this species. Our study provides the first description of reproductive manipulation by Wolbachia in a social insect and presents a new system for studying the ecological and evolutionary dynamics of host-Wolbachia associations.

\section{Results UNIDIRECTIONAL INCOMPATIBILITY}

We verified the results from Schrempf et al. (2015) by crossing queens and males from laboratory colonies of Brazilian (BR) and Japanese (JP) populations collected in 2009 and 2010, respectively (Schrader et al. 2014). We conducted this experiment once in 2015 with the same outcome (Fig. S1) and again in 2017/2018, the results of which are presented here. As expected, compared to all other crosses, matings between Brazilian queens and Japanese males resulted in lower productivity as estimated by mean weekly egg number over the first 6 weeks after initiation of egg laying, regardless of whether the male was infected with Cand. Westeberhardia or not (Fig. 1A; Kruskal-Wallis rank sum test, $\chi^{2}=78.04$, df $=6, P<0.001$; see Table $\mathrm{S} 1$ for Bonferroni-Holm corrected pairwise $P$-values). Similar results were obtained when maximum weekly egg numbers were compared (Fig. S2; KruskalWallis rank sum test, $\chi^{2}=81.10, \mathrm{df}=6, P<0.001$; see Table $\mathrm{S} 2$ for Bonferroni-Holm corrected pairwise $P$-values). Inferred hatching rates of eggs produced by crosses between Brazilian queens and Japanese males were also significantly lower, with 

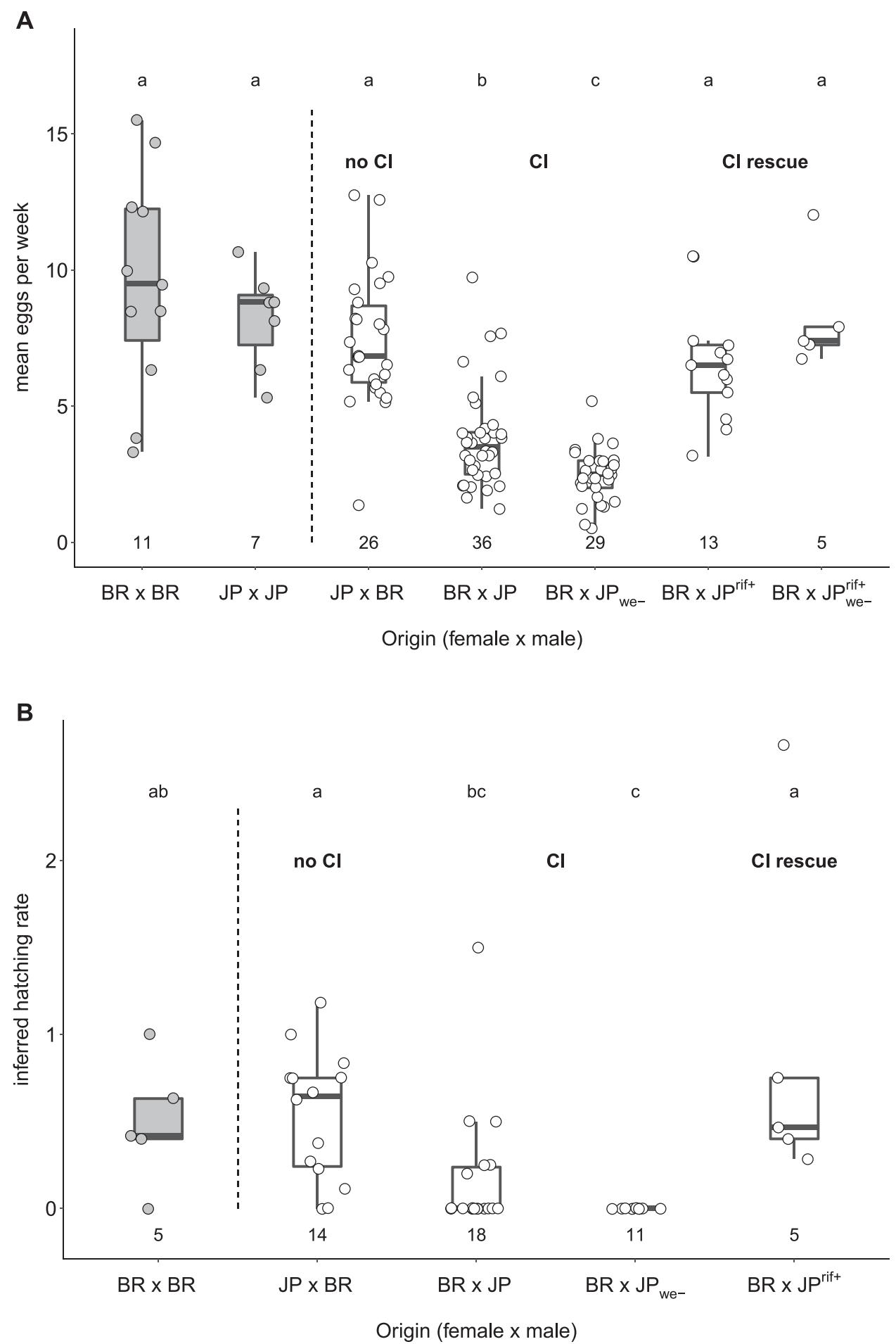

Figure 1. Mean weekly numbers and inferred hatching rates of eggs produced by intra- and interpopulation crosses of the ant $C$. obscurior. Mean weekly numbers (A) and inferred hatching rates (B) of eggs produced by Brazilian (BR) and Japanese (JP) queens mated to males from their own or from a different population. JP ants were collected from colonies that either carried the main endosymbiont Candidatus Westeberhardia cardiocondylae (JP) or did not (JP we-). Males used in "Cl rescue" crosses were collected from JP colonies with and without Cand. Westeberhardia cardiocondylae, which had been treated with the antibiotic rifampicin (JPrif+; $\mathrm{JP}_{\text {we- }}{ }^{\text {rif }}+$ ). Numbers below box plots indicate the number of replicates. Differences between groups were tested with pairwise Mann-Whitney $U$-tests followed by Bonferroni-Holm correction of $\boldsymbol{P}$-values. Letters above boxplots indicate statistically significant differences at $\boldsymbol{P}<0.05$. For Bonferroni-Holm corrected pairwise $P$-values, see Tables S1 and S3. 


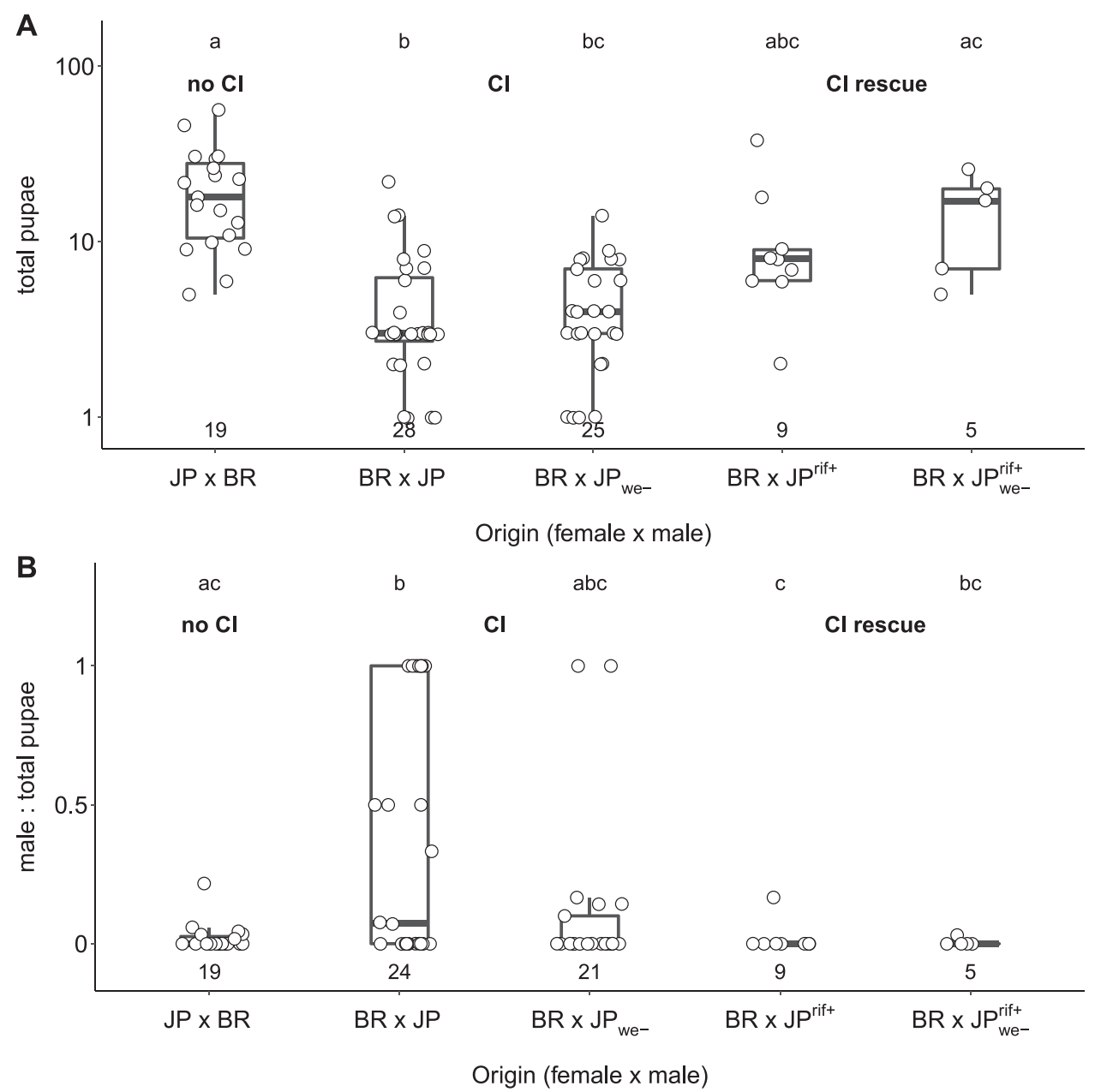

Figure 2. Total pupae numbers and pupae sex ratios produced by intra- and interpopulation crosses of the ant $C$. obscurior. Total number of pupae (A) and sex ratio of pupae (B) produced in crosses between Brazilian (BR) and Japanese (JP) queens and males over a period of 12 weeks. JP ants were collected from colonies that either carried the main endosymbiont Candidatus Westeberhardia cardiocondylae (JP) or did not ( $\left.\mathrm{JP}_{\text {we- }}\right)$. Males used in "Cl rescue" crosses were collected from JP colonies with and without Cand. Westeberhardia cardiocondylae, which had been treated with the antibiotic rifampicin (JPrif+; $\mathrm{JP}_{\text {we- }}{ }^{\text {rif }+}$ ). Numbers below box plots indicate the number of replicates. Total pupae numbers are plotted as $\log +1$ for better visualization, and differences between groups were tested with pairwise Mann-Whitney $U$-tests followed by Bonferroni-Holm correction of $\boldsymbol{P}$-values. For Bonferroni-Holm corrected pairwise $\boldsymbol{P}$-values, see Table S3. Sex ratios produced by different crosses were compared with a generalized linear model with logit link followed by manual Bonferroni-Holm correction of $\boldsymbol{P}$-values. For Bonferroni-Holm corrected pairwise $\boldsymbol{P}$-values, see Table $\mathbf{S} 4$. Letters above boxplots indicate statistically significant differences at $P<0.05$.

$58.9 \%$ (23/39) of crosses between Brazilian queens and Japanese males failing to produce larvae compared to only $12.5 \%(3 / 24)$ of crosses in the other mating combinations (Fig. 1B; KruskalWallis rank sum test, $\chi^{2}=25.43, \mathrm{df}=4, P<0.001$; see Table S3 for Bonferroni-Holm corrected pairwise $P$-values). These effects were even stronger when using males naturally uninfected with Cand. Westeberhardia, ruling out the possibility that this symbiont plays a role in causing $\mathrm{CI}$.

We monitored a subset of interpopulation crosses for an additional 6 weeks (12 weeks in total). Of the $53 \mathrm{BR} \times \mathrm{JP}$ colonies that were monitored for 12 weeks, nine failed to produce diploid offspring (queen/worker pupae), and eight produced no pupae at all. We dissected the spermathecae of five of the nine male-only-producing queens, and all contained sperm. All of the $\mathrm{JP} \times \mathrm{BR}$ colonies that were monitored for 12 weeks produced pupae, and they produced more pupae than BR $\times$ JP colonies, irrespective of whether males were infected with Cand. Westeberhardia (Fig. 2A; Kruskal-Wallis rank sum test, $\chi^{2}=39.80$, df $=4, P<0.001$; see Table S4 for Bonferroni-Holm corrected pairwise $P$-values). Furthermore, sex ratios of produced pupae were more male-biased in $\mathrm{BR} \times \mathrm{JP}$ colonies compared to JP $\times$ BR colonies (Fig. 2B; Generalized linear model with binomial error structure: $T_{4,78}=38.59, P<0.001$; see Table $\mathrm{S} 5$ for Bonferroni-Holm corrected pairwise $P$-values), an effect 
caused entirely by the lack of diploid, that is, female, pupae (Fig. S3; Kruskal-Wallis rank sum test, $\chi^{2}=5.61$, df $=4, P$ $=0.23$ ). Together, these data show consistent incompatibility between Brazilian queens and Japanese males.

\section{INCOMPATIBILITY RESCUE}

A necessary test to verify CI is "curing" the host of the putative CI-causing bacteria using antibiotic treatment, which should result in the "rescue" of the wild-type phenotype.

We fed Japanese colonies the antibiotic rifampicin diluted in honey every other week for 10 weeks, which resulted in the complete loss of the main endosymbiont Cand. Westeberhardia (Fig. S4; Mann-Whitney $U$-test, $W=34$, df $=1, P<0.001$ ) and a significant reduction of Wolbachia levels in workers (Fig. S4; Mann-Whitney $U$-test, $W=95$, df $=1, P<0.01$ ). Treating colonies with the antibiotic tetracycline did not have a negative effect on Wolbachia (Fig. S5; Mann-Whitney $U$-test, $\chi^{2}$ $=24$, df $=1, P=0.052$ ). Although Cand. Westeberhardia is permanently eradicated in subsequent generations, the negative effect of rifampicin on Wolbachia is transient, with levels returning to normal 6 months after termination of treatment (Fig. S6; Cand. Westeberhardia: Mann-Whitney $U$-test, $W=64, \mathrm{df}=1$, $P<0.001$; Wolbachia: Mann-Whitney $U$-test, $W=45, \mathrm{df}=1$, $P=0.19)$. Five of 28 rifampicin-treated colonies succumbed to the treatment and died during the 10-week period, whereas all 28 control colonies survived.

Mating Japanese males collected shortly after ceasing antibiotic feeding with Brazilian queens resulted in significantly rescued productivity, although mean and maximum weekly egg numbers did not quite reach levels of inbred queens (Fig. 1; Table S1). This rescue effect was evident regardless of whether antibiotic-treated Japanese males came from Cand. Westeberhardia-infected or uninfected colonies. Rescued colonies also exhibited higher egg hatching rates (Fig. 1B) and produced more pupae over a 12 -week period than their nonrescued counterparts (Fig. 2A), whereas sex ratios among pupae were as female-biased as in reciprocal crosses between Japanese queens and Brazilian males (Fig. 2B). Strongly reduced Wolbachia levels in antibiotic-treated Japanese males (Fig. 3; MannWhitney $U$-test, $W=163$, df $=1, P<0.001$ ) support the role of Wolbachia in inducing hybrid incompatibility.

We confirmed unidirectional CI with males from laboratory colonies of a second Asian population collected in Taiwan (TW), which is infected with a Wolbachia strain that amplifies a wsp sequence (coding for the hypervariable Wolbachia surface protein) identical to those from Japanese samples (Fig. S7). We set up crosses with queens from Brazil $(B R \times T W)$ and Japan $(\mathrm{JP} \times \mathrm{TW})$, and the reciprocal combination $(\mathrm{TW} \times \mathrm{JP})$. Again, only the combination BR $\times$ TW resulted in reduced productivity, both when mean weekly egg numbers (Fig. S8;

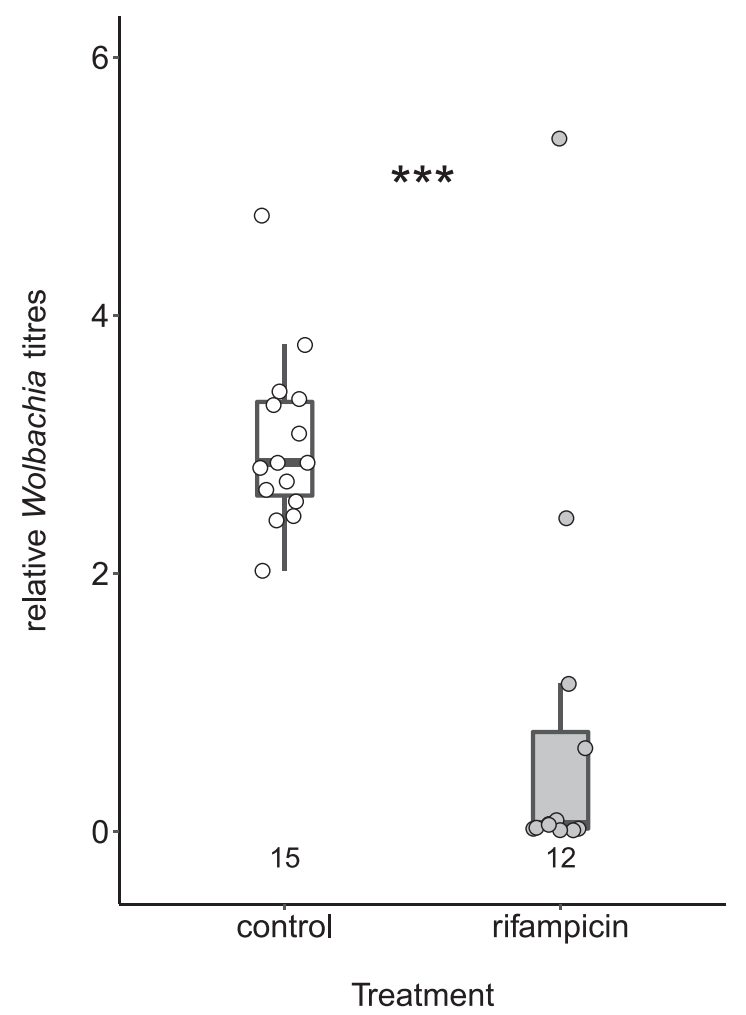

Figure 3. Effects of rifampicin treatment on Wolbachia titers in males. All males were collected from colonies that carried the main endosymbiont Candidatus Westeberhardia cardiocondylae. Numbers below box plots indicate the number of replicates. Differences in Wolbachia titers between control and rifampicin-treated males were tested with a Mann-Whitney $U$-test. Stars $(* * *)$ indicate statistically significant differences at $P<0.001$.

Kruskal-Wallis rank sum test, $\chi^{2}=9.96$, $\mathrm{df}=2, P<0.01$; see Table S6 for Bonferroni-Holm corrected pairwise $P$-values) and maximum weekly egg numbers over 6 weeks (Fig. S8; KruskalWallis rank sum test, $\chi^{2}=10.19$, df $=2, P<0.01$; see Table $\mathrm{S} 7$ for Bonferroni-Holm corrected pairwise $P$-values) were compared.

\section{Wolbachia GENOMICS AND PHYLOGENY}

We assembled the Wolbachia genomes from Brazilian ( $w$ CobsBR) and Japanese (wCobs-JP) populations of $C$. obscurior using Illumina HiSeq2000 100-bp reads of 200- and 500-bp insert paired-end libraries (Schrader et al. 2014). The genomes of Wolbachia were assembled to 148 (Brazil) and 182 (Japan) scaffolds (Table 1). Both strains showed similar genome sizes and $\mathrm{G}+\mathrm{C}$ contents, with an estimated 1013 (Brazil) and 1058 (Japan) predicted protein-coding genes. An analysis on shared genes revealed that both strains share a core of 907 proteins, with 85 unique to $w$ Cobs-BR and 109 unique to $w$ Cobs-JP. According to Bayesian phylogenetic analyses of ribosomal proteins from 23 Wolbachia strains, both Wolbachia strains belong to supergroup 
Table 1. Wolbachia genome assembly statistics. Genome assembly and draft annotation statistics for the newly sequenced Wolbachia strains from C. obscurior ( $w$ Cobs-BR and $w$ Cobs-JP). Mbp $=$ megabase pair. CDSs $=$ coding sequences. tRNAs $=$ transfer RNAs. rRNAs $=$ ribosomal RNAs. ncRNAs= non-coding RNAs.

\begin{tabular}{lll}
\hline & Wolbachia & \\
\cline { 2 - 3 } & wCobs-BR & wCobs-JP \\
\hline Assembled genome size (Mbp) & 1.19 & 1.30 \\
Number of scaffolds & 148 & 182 \\
N50 & 14,698 & 16,173 \\
G + C content (\%) & 35.3 & 35.1 \\
Predicted CDSs* & 1013 & 1058 \\
tRNAs & 34 & 34 \\
rRNAs & 3 & 3 \\
Other ncRNAs genes/motifs & 71 & 54 \\
\hline
\end{tabular}

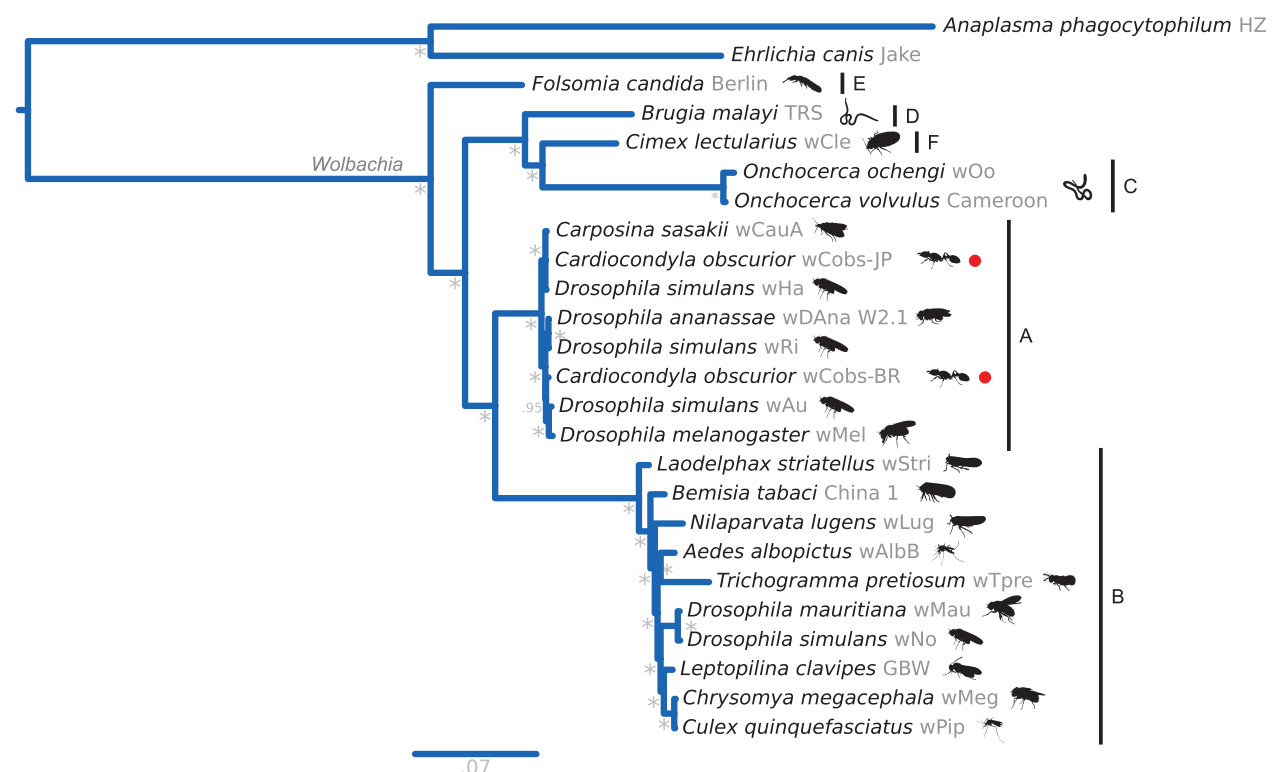

Figure 4. Wolbachia spp. phylogeny. Bayesian phylogenetic placement of Wolbachia strains sequenced from C. obscurior (wCobs-BR and wCobs-JP). Anaplasma phagocytophilum and Ehrlichia canis were used as outgroups. For Wolbachia, names at tips correspond to the host from which they were sequenced/isolated. Red dots are used to highlight the newly sequenced strains. Next to the binomial names, strain names are shown in gray and silhouettes for the host are sketched in black. Horizontal bars delimit Wolbachia supergroups (A-F). $*=1.0$ posterior probability.

A, which together with supergroup B contains strains capable of reproductive manipulation. $w$ Cobs-JP was found to be more closely related to $w \mathrm{Ha}$ and $w \mathrm{CauA}$ strains, whereas $w$ Cobs-BR was phylogenetically closer to $w \mathrm{Au}$ and $w \mathrm{Mel}$ (Fig. 4).

\section{MECHANISM}

CI usually results in the failure to produce viable zygotes but how CI operates functionally is still not clear. The two championed models are based on (1) a sperm modification step during spermatogenesis that is reversed by a rescue factor or (2) the transfer of a toxic product via the sperm and subsequent binding to a female-derived antidote (Shropshire and Borden- stein 2019; Shropshire et al. 2019; Beckmann et al. 2019a,b). The current genetic view for CI in Drosophila favors a twoby-one model (Shropshire et al. 2019), involving two adjacent genes (coined cifA and cifB) located in a region of prophage origin in $w$ Mel (Beckmann et al. 2017; LePage et al. 2017; Shropshire et al. 2018; Chen et al. 2019; Shropshire and Bordenstein 2019; Shropshire et al. 2019), which contains several genes with eukaryote function (Perlmutter et al. 2019). Expression of both cifA and cifB in males is required to cause CI, whereas expression of cifA rescues embryo mortality (Shropshire et al. 2018). Phylogenetic analyses of the Cif proteins have revealed at least five distinct phylogenetic clades referred to as 

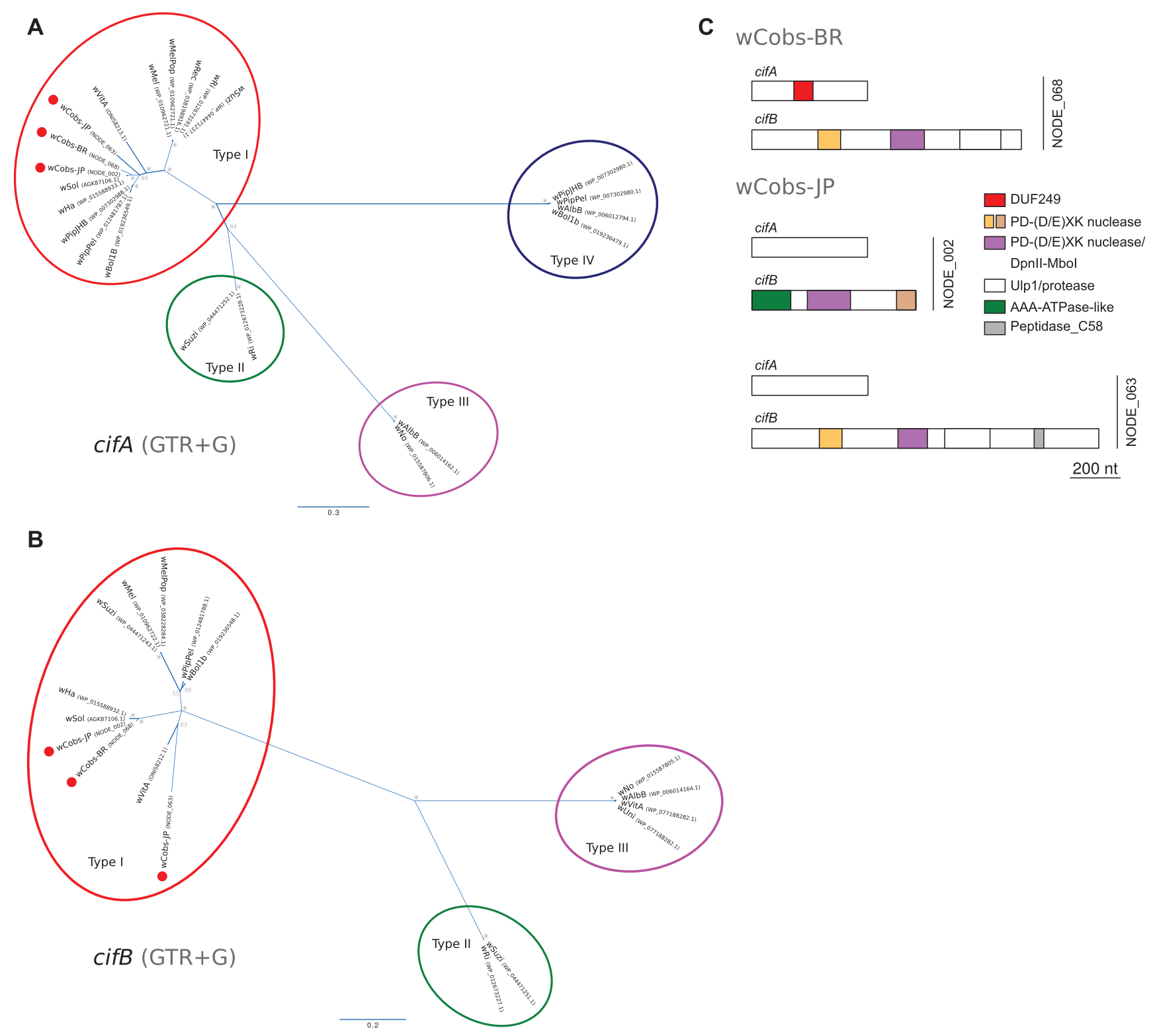

Figure 5. Phylogeny and domain structure of cif genes. Bayesian phylogenetic trees for (A) cifA and (B) cifB genes. Colored ovals are used to highlight the different gene "types." * $=1.0$ posterior probability. (C) Domain structure for the cif genes of wCobs-BR (top) and wCobs-JP (bottom) strains. For the latter, the two loci are shown and indicated with a vertical bar to the right of the sketches. Color codes and designation of the domains are shown in the legend.

Types 1-5 (LePage et al. 2017; Lindsey et al. 2018; Bing et al. 2020).

We found homologues of cifA and cifB in Wolbachia strains from Brazil and Japan. In the latter, we found two additional homologues. Through Bayesian phylogenetic inference, and following the classification scheme of Lindsey et al. (2018), we found that all copies of cifA and cifB belong to Type I (Fig. 5A, B). The cifA and cifB genes found in NODE_002 of $w$ Cobs-JP are closely related to those found in $w$ Cobs-BR and in the cryptic phage of the strain $w$ Sol isolated from the fig wasp Ceratosolen solmsi (Fig. 5A, B). The cifA and cifB genes found in NODE_063 of $w$ Cobs-JP show a more distant relationship to their homologues in $w$ Cobs-BR. Following Lindsey et al. (2018), we performed a protein remote homology search with the web server of HHpred version 3.2.0 (Zimmermann et al. 2018). Although the cifA gene of $w$ Cobs-BR contained a DUF249 domain, neither cifA homologues in $w$ Cobs-JP showed homology to known domains (Fig. 5C). In contrast, putative domains were found in all cifB genes. These shared a putative nuclease domain (PD-(D/E)XK nuclease/DpnII-MboI) found in other Type I cifB genes. The cifB homologue found in NODE_063 in $w$ Cobs-JP shared most domains with the $c i f B$ homologue in 

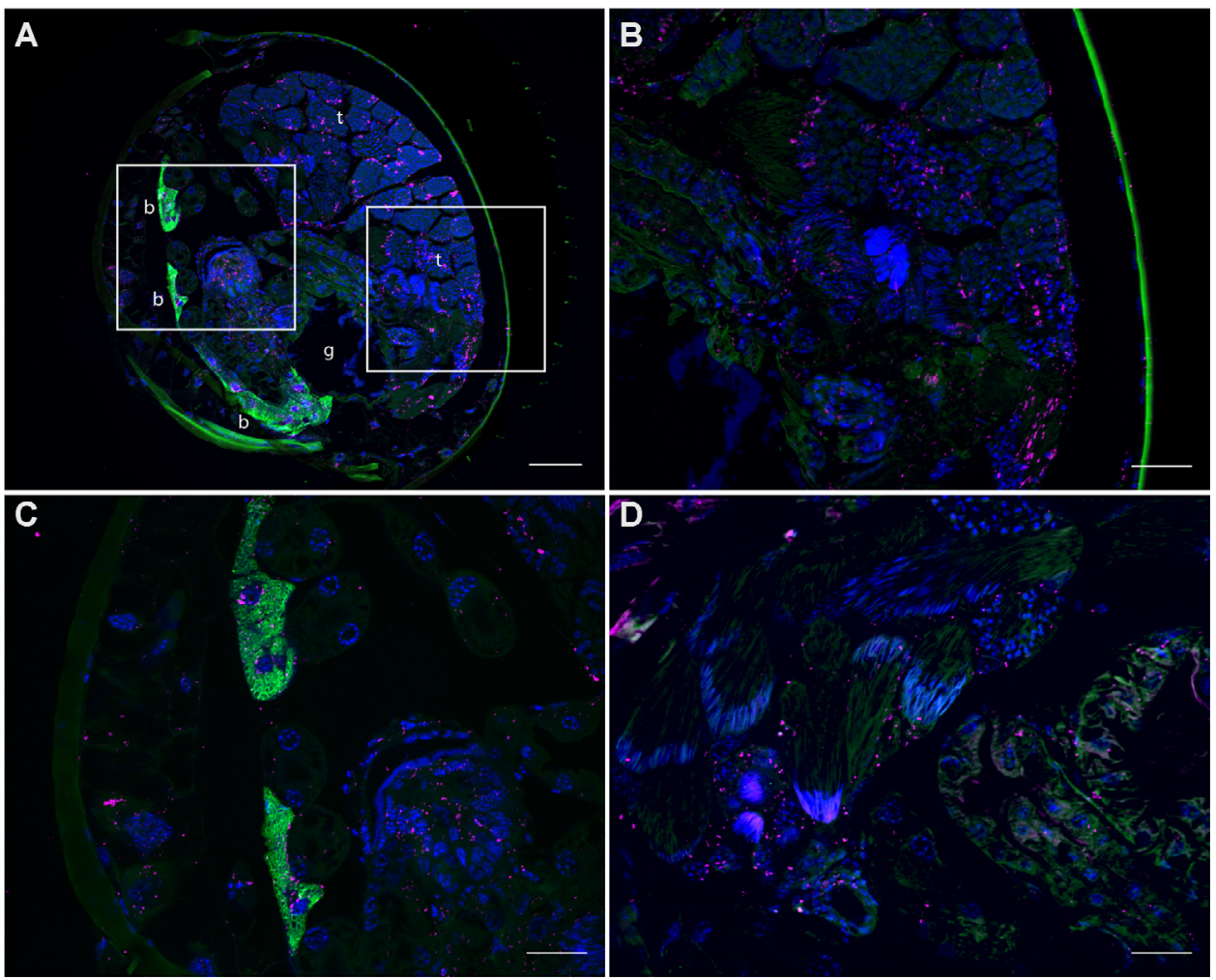

Figure 6. Localization of endosymbiotic bacteria in male adult abdomens of $C$. obscurior via fluorescence in situ hybridization. Wolbachia were specifically stained with two Cy5-labeled probes (magenta) and Cand. Westeberhardia with a Cy3-labeled probe (green). Host cell nuclei are counterstained with DAPI (blue). (A) Transversal section of C. obscurior JP male abdomen showing the testes ( $t$ ), the Cand. Westeberhardia-containing bacteriomes (B) and the gut (g). The areas indicated by rectangles are displayed in higher magnification showing Wolbachia-infected seminiferous tubules (B) and bacteriomes co-infected with Cand. Westeberhardia and Wolbachia (C). (D) Cardiocondyla obscurior BR male testes and gut epithelium infected with Wolbachia. Scale bars: $50 \mu \mathrm{m}$ (A) and $20 \mu \mathrm{m}$ (B-D).

$w$ Cobs-BR, with an additional peptidase domain (Peptidase_C58; PF03543).

\section{Wolbachia INFECTION DYNAMICS}

To compare infection titers between populations, we performed qPCR of the Wolbachia gene cytochrome c oxidase subunit 1 ( $\operatorname{coxA}$ ) against the ant housekeeping gene elongation factor 1 alpha 1 (EF1). Japanese queens had higher relative $w$ Cobs-JP titers than Brazilian queens infected with $w$ Cobs-BR (Fig. S9; Mann-Whitney $U$-test, $W=23$, df $=1, P=0.043$ ). Likewise, Japanese workers showed higher infection titers with $w$ Cobs-JP compared to Brazilian workers infected with $w$ Cobs-BR (Fig. S9; Mann-Whitney $U$-test, $W=16$, df $=1, P=0.017$ ).

To test for morph bias in infection, we compared coxA levels in adult queens, workers, winged males, and wingless males using qPCR. coxA levels did not differ between sexes and were consistently higher in winged morphs (queens, winged males) compared to wingless morphs (workers, wingless males) (Fig. S10; Kruskal-Wallis rank sum test, $\chi^{2}=19.53$, df $=3, P<0.001$ ).

To measure the potential cost of Wolbachia infection and to test for a selective loss in workers, we compared infection levels of workers and queens over time. In contrast to Cand. Westeberhardia (Klein et al. 2015), Wolbachia levels quickly reached a plateau in both queens and workers. There was no indication that individuals lose Wolbachia over the first few weeks of their lives, which typically last for up to 3 (workers) or 6 months (queens) (Fig. S11; workers: ANOVA, $F_{4,26}=2.69, P=0.059$; queens: ANOVA, $\left.F_{5,50}=15.95, P<0.001\right)$.

\section{Wolbachia LOCALIZATION IN MALES}

Wolbachia-induced CI has been linked to sperm modification during spermatogenesis (Veneti et al. 2003) or the transfer of a CI toxin along with or in the sperm. The presence of Wolbachia in the testes is thus expected, although their specific localization in spermatocytes or spermatids might not be a prerequisite for inducing CI (Clark et al. 2008). We used fluorescence in situ hybridization to localize Wolbachia in the abdomens of male C. $o b$ scurior, confirming the presence of the bacteria throughout different tissues including the testes in both Japanese (Fig. 6A-C) and Brazilian (Fig. 6D) individuals. Wolbachia was also observed co-infecting bacteriocytes dominated by Cand. Westeberhardia, yet in significantly lower densities (Fig. 6C). 


\section{Discussion}

CI results in embryonic death because of symbiont-induced incompatibilities between oocytes and sperm. In species with haplodiploid sex determination such as ants, in which females develop from fertilized, diploid eggs and males develop from unfertilized, haploid eggs, CI is furthermore expected to result in male-biased offspring sex ratios (Vavre et al. 2000; Egas et al. 2002). In the ant $C$. obscurior, we found a decrease in numbers and inferred hatching rates of eggs, as well as a drastic reduction in pupae production and an increase in male-biased sex ratios in colonies founded by Brazilian queens mated to Japanese males. In contrast, there were no effects on productivity and sex ratios in "rescued" crosses between Brazilian queens and antibioticcured Japanese males, nor in reciprocal crosses between Japanese queens and Brazilian males or in intrapopulation crosses. A previous study emphasized the role of Bateson-Dobzhansky-Muller incompatibilities, in which two parental loci that have diverged cause hybrid sterility or inviability, in causing a CI-like phenotype in C. obscurior (Schrempf et al. 2015). Wolbachia-induced incompatibilities were ruled out because of identity of a partial sequence of wsp extracted from seven individuals (three from Brazilian colonies collected in 2004 and four from Japanese colonies collected in 2005) using the wsp81F/wsp691R primer combination (Braig et al. 1998). Gene expression comparisons of allopatrically and sympatrically mated Brazilian queens pointed toward elevated immune responses and other changes that mimicked a CI-like phenotype (Schrempf et al. 2015). Based on the results of the current study, a more parsimonious explanation for these effects is a classic unidirectional CI mechanism induced by the Japanese but not the Brazilian Wolbachia strain, which acts after fertilization and leads to the death of diploid, female embryos. That $\mathrm{CI}$ in C. obscurior affected both egg hatching rate (the classical measure of $\mathrm{CI}$ ) and overall egg numbers is probably because ants rear their brood cooperatively and developing individuals depend on nestmate care. Ant workers display excellent discrimination abilities and can discriminate between eggs depending, for instance, on their viability, sex, or colony origin (Schultner and Pulliainen 2020). Eggs were counted once per week in our experiment; with an egg developmental duration of 5-8 days (Schrempf and Heinze 2006; Schrader et al. 2015), efficient detection and removal of unviable eggs by workers in colonies from CI crosses likely resulted in lower weekly egg numbers.

The genetic mechanism of CI in C. obscurior appears to align along the Wolbachia cif gene axis described in Drosophila fruit flies and Culex mosquitoes (Beckmann et al. 2017, 2019b; Bonneau et al. 2018; Lindsey et al. 2018; Shropshire et al. 2019). This genetic region is of prophage origin and capable of manipulating the eukaryote host (LePage et al. 2017), showcasing the importance of using a hologenomic approach when studying evolution (Brucker and Bordenstein 2013). Similar prophage-derived regions containing pairs of homologue ciflcif-like genes have since been described in a number of Wolbachia strains (Lindsey et al. 2018; Bing et al. 2020; Martinez et al. 2021). Surprisingly, the homologues of $c i f B$ in $w$ Pip and $w$ Mel have different biochemical functions: both putatively contain ankyrin-like repeats for protein-protein interaction with eukaryotic DNA (Bonneau et al. 2018) but cif $B_{\mathrm{wMel}}$ is not a putative deubiquitinase (Shropshire and Bordenstein 2019), as postulated for cifB $B_{\mathrm{wPip}}$ (Beckmann et al. 2017). Both Wolbachia strains isolated from $C$. obscurior have homologues of cifA and cifB but only the $w$ CobsJP strain has an additional $c i f A / B$ pair. This could point toward a role for the latter pair in inducing $\mathrm{CI}$, perhaps due to functional differences in $c i f A / B$ homologues between $w$ Cobs-JP and $w$ Cobs-BR, or because of an interaction of the two cif gene products in Japanese males. Whether the second cifA/B-containing region in $w$ Cobs-JP stems from a duplication event and subsequent divergence (Beckmann et al. 2019b) or from horizontal transfer from anotherWolbachia strain (Martinez et al. 2021) remains to be shown.

Wolbachia clearly play a significant role in the biology of the ant host, with all screened individuals infected regardless of population, caste, or sex. Similar infection patterns have been found in the invasive yellow crazy ant Anoplolepis gracilipes (Lee et al. 2020). In contrast, Wolbachia infection is facultative in the closely related invasive ant Monomorium pharaonis (Pontieri et al. 2017). Wolbachia titers were overall higher in individuals from Japanese colonies infected with $w$ Cobs-JP, which may be linked to the strain's ability to induce CI (Breeuwer and Werren 1993; Perrot-Minnot and Werren 1999). Wolbachia titers were also higher in queens and winged males compared to workers and wingless males, a difference that is likely associated with the relative amounts of reproductive tissue as $C$. obscurior workers lack ovaries and winged males have larger testes than wingless males (Schrempf et al. 2016). In addition, rifampicin treatment merely reduced Wolbachia titers, and this effect was reversible. This was not the case for Cand. Westeberhardia, which was permanently cleared by rifampicin treatment. Also, Wolbachia titers did not decline with age in workers. Wolbachia infection can be costly and selection may thus favor reduced infection loads in sterile social insect workers, as these do not contribute to vertical transmission (Wenseleers et al. 2002; Treanor et al. 2018). This has been shown to occur in Acromyrmex leaf cutter ant workers (Andersen et al. 2012). Such a mechanism also appears to be acting on Cand. Westeberhardia, where titers in workers but not queens decreased significantly with age, indicating that although the host may benefit from infection during development or in the adult egg-laying queen stage, the symbiont is not required (Klein et al. 2015). In contrast, consistently high densities of Wolbachia in C. obscurior workers suggest that infection is not costly in this species, and that the bacteria may even have positive effects 
on the fitness of their ant host. One possibility is that Wolbachia plays a role in nutritional provisioning (Cheng et al. 2019). Apart from CI, the two Wolbachia strains do not appear to have strongly differing effects on host fitness under controlled laboratory conditions; however, whether a strain confers benefits in natural conditions, as well as whether Wolbachia infection itself influences fitness, remains to be studied.

Our study provides important clues for understanding the evolutionary history of the C. obscurior-Wolbachia association. The ant probably originated in Southeast Asia, with drift events leading to genetic differentiation in subpopulations and subsequent introduction to the New World. Sequence identity between Wolbachia strains isolated from populations collected in Japan and Taiwan indicates that an association with $w$ Cobs-JP is ancestral in this species, and that acquisition of the new strain occurred prior to or after invasion of the New World. The latter scenario is more parsimonious, as geographic patterning separating New from Old World Wolbachia strains has been previously observed in ants (Kautz et al. 2013), and frequent horizontal transmissions (Russell et al. 2009; Tolley et al. 2019) can lead to surprising diversity within species (Kelly et al. 2019). Cardiocondyla obscurior and its sister species $C$. wroughtonii are the only known representatives of the $100+$ described species in the genus Cardiocondyla that have adopted an arboreal lifestyle, as opposed to nesting in soil and rock crevices. This makes them highly susceptible to horizontal transmission of Wolbachia from other plantassociated insects. Once acquired, selective advantages conferred by the novel strain in the new environment may have helped the strain persist (Versace et al. 2014).

Global exchange of stowaway ants leading to secondary contact between allopatric populations is common in ants, for example, in the tropical fire ant Solenopsis geminata (Gotzek et al. 2015) and the little fire ant Wasmannia auropunctata (Foucaud et al. 2010). Thus, the presented scenario, albeit conceived in the laboratory, is not an unrealistic one. Generally, unidirectional CI is thought to facilitate introgression between populations (Shoemaker et al. 1999; Jaenike et al. 2006; Cooper et al. 2017), whereas bidirectional CI should promote divergence (Bordenstein and Werren 2007; Brucker and Bordenstein 2012). In theory, gene flow can be reduced in both scenarios (Telschow et al. 2005; Telschow et al. 2007). How easily a CI-inducing Wolbachia strain can spread through a population depends on its transmission rate to offspring, the level of CI mortality it induces, and its general effects on female fecundity (Engelstädter and Telschow 2009). Transmission rates of Wolbachia in C. obscurior appear to be high because all screened individuals were infected, whereas embryonic mortality caused by $w$ Cobs-JP was moderate at $\sim 60 \%$ and effects on female fecundity do not seem to differ between the two strains. This predicts mixed $C$. obscurior genotypes but primarily the presence of the $w$ Cobs-JP strain in secondary con- tact zones, assuming that $\mathrm{JP} \times \mathrm{BR}$ hybrids perform as well as parental lineages. If Wolbachia infection has additional fitness effects, either because it is costly (Hoffmann et al. 1996; Vala et al. 2000; Perrot-Minnot et al. 2002) or because the bacteria provide the host with benefits such as nutrient provisioning (Hosokawa et al. 2010) or pathogen resistance (Moreira et al. 2009), selection on specific host-Wolbachia associations may further modulate these dynamics. In addition, infection dynamics may be influenced by host population structure and traits such as haplodiploidy and level of inbreeding (Branca et al. 2009; Engelstädter and Telschow 2009). Like in many invasive ants, colonies of $C$. obscurior are headed by several queens and disperse by colony budding, which occurs when groups of queens and workers leave the nest on foot to found a new colony nearby. Prior to dispersal, virgin queens and males can mate in the nest; in $C$. obscurior, matings readily occur between siblings (Heinze 2017) and even between mothers and sons (pers. obs.), leading to high levels of inbreeding. The resulting high viscosity of $C$. obscurior populations may contribute to the maintenance of infection polymorphisms in secondary contact zones. Indeed, the Japanese strain occurs in some populations in Brazil (Klein 2015). A global genomic survey of Wolbachia-C. obscurior associations is currently underway, which promises to provide further insight into the invasion history of the ant (Tseng et al. 2019), and into dynamics of CI in this system. Coupled with experiments assessing the fitness of different host genotype $\times$ Wolbachia combinations under varying environmental conditions, and genomic and phenotypic characterization of hybrid offspring, this will help unravel how the symbiosis helps shape the evolution of $C$. obscurior and contribute to a better understanding of symbiont-driven evolutionary dynamics in tramp and invasive species.

\section{Methods}

Cardiocondyla obscurior presumably originated in Southeast Asia and is now distributed around the tropics and subtropics. Queens in C. obscurior are produced year-round and have a short generation time of $\sim 120$ days (from egg to queen to egg). Colonies usually contain two to five queens (Schrader et al. 2014) and a single wingless male that can live up to a few months and continually produces sperm (Heinze and Hölldobler 1993). Such males transfer about 1000 sperm cells per copulation (Schrempf et al. 2007), which is sufficient for a lifelong production of diploid offspring by an inseminated queen. Colonies may also periodically produce winged males, which differ from wingless males in their morphology and reproductive physiology (Heinze and Hölldobler 1993). Newly hatched queens can decide to stay in the maternal nest or to leave-alone or with a small group of workers-irrespective of mating status. Like all Hymenoptera, 
ants exhibit haplodiploid sex determination and virgin females can produce males from haploid, unfertilized eggs. Virgin queens in C. obscurior can copulate with their brothers (Heinze 2017) and own sons (pers. obs.); this also occurs in another Cardiocondyla species (Schmidt et al. 2013). This biology has facilitated the worldwide spread of several species of the genus (Heinze et al. 2006).

In this study, we used inbred laboratory "populations" derived from one colony each collected in Bahia, Brazil (BR) in 2009 and Okinawa, Japan (JP) in 2010 (Schrader et al. 2014). The JP population exhibits a natural polymorphism regarding the presence of the endosymbiont Cand. Westeberhardia cardiocondylae, with some colonies being naturally uninfected (JP $\left.{ }_{\text {we- }}\right)$ (Klein et al. 2015). The populations from Brazil and Japan show remarkable genomic differentiation, with $553 \mathrm{k}$ single nucleotide variants between the two populations in a $187.5 \mathrm{MB}$ draft genome. They also differ in cuticular hydrocarbon composition, number of queens per colony, queen size, and behavior (Schrader et al. 2014). However, workers are morphologically indiscernible according to exploratory data analyses by hierarchical and nonhierarchical, vector-based forms of Nest Centroid (NC) clustering: NC-Ward, NC-part.hclust, NC-part.kmeans, and NC-NMDS-kmeans (Seifert et al. 2013, 2017). These analyses considered 15 continuous morphometric characters. Specifically, when considering 82 cosmopolitan nest samples of the sister taxa $C$. wroughtonii and C. obscurior, all JP and BR samples were allocated to the latter species by all four types of $\mathrm{NC}$ clustering. This result was confirmed with posterior probabilities of $P>0.98$ if these samples were run as wild cards in a linear discriminant analysis. Analyses of 32 nest samples representing only the $C$. obscurior cluster could not allocate the JP and BR populations to different intraspecific clusters nor could these analyses demonstrate any intraspecific structuring at all. This suggests that there is, of yet, no divergence into morphospecies. In addition to JP and BR colonies, we used colonies collected from a second Asian population in Taipei, Taiwan in 2013 (TW) to confirm the presence of unidirectional CI.

For all experiments, colonies were kept in square plasterbottom nests $(100 \times 100 \times 20 \mathrm{~mm}$; Sarstedt, Germany) and held in climate chambers under a $12 \mathrm{~h} / 12 \mathrm{~h}$ light/dark cycle and a $28^{\circ} \mathrm{C} / 22^{\circ} \mathrm{C}$ temperature cycle. Food (honey and Drosophila or pieces of Periplaneta americana) and water were provided to stock colonies every 3 days. All animal treatment guidelines applicable to ants under international and German law were followed. Collection of colonies that form the basis of the laboratory population used in this study was permitted by the Brazilian Ministry of Science and Technology (permits 20324-1/40101-1). No other permits were required for this study.

\section{ANTIBIOTIC TREATMENT}

Rifampicin is a broad-spectrum antibiotic that acts via inhibition of bacterial RNA polymerase (Campbell et al. 2001). To produce Wolbachia-cured wingless males, we split large Cand. Westeberhardia-infected (JP) and uninfected ( $\left.\mathrm{JP}_{\text {we- }}\right)$ stock colonies into two equal halves. Half of these splits were designated as controls (JP: $n=13$; $\mathrm{JP}_{\mathrm{we}-}: n=5$ ), whereas the other half was treated with antibiotics $\left(\mathrm{JP}^{\text {rif }+}: n=13 ; \mathrm{JP}_{\mathrm{we}-}{ }^{\text {rif }}{ }^{+}: n=5\right)$. We diluted $0.0025 \mathrm{~g}$ of solid rifampicin (Sigma-Aldrich, USA) in $500 \mu \mathrm{L}$ of a 1:1 honey-water solution for a final antibiotic concentration of $5 \mu \mathrm{g} / \mu \mathrm{L}$. The antibiotic solution was placed on a shaker for $4 \mathrm{~h}$ to ensure complete mixing, and subsequently covered with aluminum foil and stored at $-20^{\circ} \mathrm{C}$. Treated colonies were fed with the antibiotic solution twice per week every other week, for a total duration of 10 weeks. On the days following treatment, the antibiotic solution was removed from the nest. Between antibiotic feedings (i.e., every other week), colonies were fed with honey and pieces of autoclaved cockroach (to prevent bacterial re-infections) and water ad libitum. Control colonies were fed twice a week with honey and cockroaches and received water ad libitum.

\section{EXPERIMENTAL CROSSES}

We designed seven mating combinations between queens and wingless males from the two populations: (1) JP $\times \mathrm{JP}(n=7)$, (2) $\mathrm{JP} \times \mathrm{BR}(n=26),(3) \mathrm{BR} \times \mathrm{BR}(n=11),(4) \mathrm{BR} \times \mathrm{JP}(n=$ 36), (5) $\mathrm{BR} \times \mathrm{JP}_{\mathrm{we}}(n=29),(6) \mathrm{BR} \times \mathrm{JP}^{\text {rif }+}(n=13)$, and (7) $\mathrm{BR} \times \mathrm{JP}_{\text {we- }}{ }^{\text {rif }+}(n=5)$. Each experimental colony was set up with one queen pupa, one freshly hatched wingless male, 10 workers, and brood (10 eggs, 10 larvae, and 10 worker pupae). All individuals were collected from unmanipulated stock colonies, except for $\mathrm{JP}^{\text {rif }+}$ and $\mathrm{JP}_{\text {we- }}{ }^{\text {rif }+}$ males used in combinations \#6 and \#7, which were collected from rifampicin-treated colonies. To avoid manipulation effects, all combinations were set up in parallel. In such experimental colonies, mating between queens and males usually occurs within 3 weeks, after which queens shed their wings and begin laying eggs. We monitored colonies for queen wing loss once per week. Once queens had shed their wings, we removed any brood placed in the colony during setup and standardized worker number to 20 individuals.

Colonies were monitored once a week for 6 weeks and all eggs and pupae (workers, queens, and males) counted. A subset of colonies was monitored for a total of 12 weeks $(\mathrm{JP} \times \mathrm{BR}$ : $n=19 ; \mathrm{BR} \times \mathrm{JP}: n=28 ; \mathrm{BR} \times \mathrm{JP}_{\mathrm{we}-}: n=25 ; \mathrm{BR} \times \mathrm{JP}^{\text {rif }+}$ : $\left.n=9 ; \mathrm{BR} \times \mathrm{JP}_{\text {we- }}{ }^{\text {rif }+}: n=5\right)$. In all colonies, the number of adult workers was kept constant at 20 individuals by removing hatched workers or adding workers from stock colonies. From monitoring data, we calculated mean and maximum weekly egg numbers, and total number and sex ratio of produced pupae. We also attempted to infer the hatching rates of eggs to make our 
results comparable with other studies. In C. obscurior, eggs do not survive in isolation and following individual eggs until hatching within colonies is difficult both because eggs are continuously produced and constantly moved and because frequent disturbances can stress colonies, causing workers to neglect brood. To overcome this caveat, in a subset of colonies $(\mathrm{BR} \times \mathrm{BR}: n=$ 5; JP $\times$ BR: $n=14$; BR $\times$ JP: $n=18 ; \mathrm{BR} \times \mathrm{JP}_{\mathrm{we}}: n=11$; $\mathrm{BR} \times \mathrm{JP}^{\text {rif }+} ; n=5$ ), we collected data on the number and instar of larvae produced per week over a period of $\sim 8$ weeks, allowing us to calculate a proxy for egg hatching rate:

Inferred hatching rate $=\frac{\text { First }+ \text { second instar larvae in week } 7}{\text { Eggs in week } 5}$.

Cardiocondyla obscurior eggs hatch in 5-8 days (Schrempf and Heinze 2006) and the approximate durations of the first and second larval instars are 3 and 5 days, respectively (Schrader et al. 2015). Thus, any eggs present in colonies in week 5 will have hatched and reached the first or second instar 2 weeks later.

To verify that $\mathrm{CI}$ is specifically induced by the $w$ Cobs-JP strain when it encounters the $w$ Cobs-BR strain, we set up three additional mating combinations between queens and males using a third population from Taiwan (TW) that also harbors the $w$ Cobs-JP strain: (1) JP $\times \mathrm{TW}(n=5),(2) \mathrm{TW} \times \mathrm{JP}(n=6)$, and $(3) \mathrm{BR} \times \mathrm{TW}(n=5)$. Experimental colonies were set up as described above and weekly egg numbers documented over a period of 6 weeks.

\section{DNA SEQUENCING AND GENOME ASSEMBLY}

For a previous study, we sequenced DNA from a pool of 26 (JP) and 30 (BR) males (Schrader et al. 2014). To extract Wolbachia sequences from these data, the reads were righttail clipped (minimum quality of 20) and all reads with undefined nucleotides were removed using a combination of FASTXToolkit version 0.0.14 (http://hannonlab.cshl.edu/fastx_toolkit/, last accessed December 3, 2010) and PRINSEQ++ version 1.2 (https://doi.org/10.7287/peerj.preprints.27553v1). The obtained paired-end reads were assembled with SPAdes version 3.13.1 (-k 33,55,77-only assembler; Bankevich et al. 2012). The resulting contigs were then binned according to an ad hoc BLASTX-based method. First, a proteome database was built from representative genomes (Table S8). Second, the best hit for each scaffold was used for assigning a preliminary bin and a coverage cutoff $(10 \times$ for BR and $30 \times$ for JP) was implemented based on the coverage of the longest contigs. Third, each scaffold was manually inspected using the online NCBI web server versus BLAST+2.9.0. If the best hits were consistently to proteins from Wolbachia bacteria across the scaffold, the query sequence was retained. Finally, the assembly graph was loaded into Bandage version 0.8.1 (Wick et al. 2015), the graph(s) matching Wolbachia sequences were identified, and the sequences were extracted. These extracted se- quences were then compared to the Wolbachia draft bin. For both JP and BR draft assemblies, the total length of the extracted sequences was roughly the same as that of the manually curated bins, adding only a couple of kilo base pairs (kbp) and thus suggesting near completeness of the genome. Using these Wolbachia bins, we performed a mapping using BOWTIE2 version 2.3.4.1 (Langmead and Salzberg 2012) followed by a reassembly of the mapped reads in SPAdes (with previously mentioned parameters). This final reassembly was filtered by both coverage and manual screening of scaffolds shorter than $3 \mathrm{kbp}$. Assembled sequences have been deposited in the European Nucleotide Archive (ENA) under the accessions CACTIU010000000.1 ( $w$ Cobs-BR) and CACTIV010000000.1 (wCobs-JP).

\section{PHYLOGENY AND COMPARATIVE GENOMICS OF Wolbachia STRAINS}

A draft annotation of the scaffolds was done using Prokka version 1.14 (Seemann 2014). For comparative genomic purposes, we built a subset of the predicted open reading frames (ORFs) by filtering out all proteins annotated as "hypothetical proteins" with genes shorter than 300 base pairs (bp). This helped us avoid wrongly annotated ORFs overlapping pseudogenes. To infer the phylogenetic relationship between Wolbachia strains found to infect the analyzed BR and JP populations as well as among other Wolbachia bacteria, we collected the amino acid sequences for the ribosomal proteins of 23 Wolbachia strains from supergroups A (8), B (10), C (2), D (1), E (1), and F (1) (Table S9). Using Anaplasma phagocytophilum strain $\mathrm{HZ}$ and Ehrlichia canis strain Jake as outgroups, we inferred a Bayesian phylogeny using MrBayes version 3.2.6 (Ronquist et al. 2012). We ran two independent analyses with four chains each for 300,000 generations and checked for convergence. The substitution model JTT $+\mathrm{I}+\mathrm{G}+\mathrm{F}$ was used as suggested by jModelTest version 2.1.10.

To infer the shared protein-coding gene content of $w$ CobsBR and $w$ Cobs-JP strains, we used OrthoMCL version 2.0.9 ( $\mathrm{Li}$ 2003; Chen et al. 2007) with an inflation value of 1.5. The synteny of both strains was assessed using nucmer version 3.23 (Kurtz et al. 2004) with anchor matches that were unique in both the reference and query (-mum).

\section{cif GENE FAMILY ANNOTATION AND PHYLOGENETICS}

cif genes were first identified by BLASTP using the protein sequences WP_010962721.1 (cifA) and WP_010962722.1 (cifB) as references. Predicted start codons were manually checked for the presence of a Shine-Dalgarno-like sequence. To identify whether the cif genes form each strain belonged to the same type or not, we performed a phylogenetic analysis following Lindsey et al. (2018). Briefly, the previously used protein sequences were aligned with the newly acquired ones from both 
wCobs-BR and $w$ Cobs-JP. The alignments were then backtranslated to nucleotides. For $c i f B$, the type IV genes were excluded and the $3^{\prime}$-end of the alignment was manually truncated to remove the longer unmatched regions of some cifB variants. Bayesian phylogenetic inference was performed with $\mathrm{Mr}-$ Bayes version 3.2.6 with the GTR + G substitution model (as suggested by jModelTest) running two independent analyses with four chains each for 1,000,000 generations, and checked for convergence. Following Lindsey et al. (2018), Cif protein domains were searched using HHpred's version 3.2.0 web server (https: //toolkit.tuebingen.mpg.de/tools/hhpred) with default parameters and the following databases: SCOPe70 version 2.07, COG/KOG version 1.0, Pfam-A version 32.0, and SMART version 6.0. Hits with a probability $\geq 80 \%$ were considered.

All genomic data files are available at the Zenodo repository https://doi.org/10.5281/zenodo.3561160.

\section{Wolbachia AND Cand. Westeberhardia INFECTION}

We assessed the presence and titers of bacterial infections using PCR and qPCR, respectively, on DNA extracted from whole bodies (worker pupae and adults, queen pupae and adults, adult males). DNA was extracted from samples using a standard CTAB protocol.

\section{PRESENCE OF INFECTION}

We confirmed infection in a subset of worker pupae from Brazilian and Japanese colonies ( $n=5$ pools of five workers from five colonies per population). Infection with Wolbachia was tested with a PCR assay using primers specific for a fragment of the Wolbachia surface protein (wsp) gene of the BR (105-bp, wsp_wCobs-BR-for: 5'-TAAATCTTGCATCTGTAACATT-3', $w s p \_w$ Cobs-BR-rev: 5'-CTGCGGATACTGATACAACTACTG$\left.3^{\prime}\right)$ and JP strains (254-bp, wsp_wCobs-JP-for: 5'CATTTTGACTACTCACAGCGGTTG-3', wsp_wCobs-JP-rev: 5'-CTGCGGATACTGATACAACTACTG-3'). Candidatus Westeberhardia infection was tested by amplifying a 204-bp fragment of the ribonucleoside-diphosphate reductase 1 subunit beta gene ( $n r d B$, WEOB_403; Klein et al. 2015). A 104-bp fragment of the $C$. obscurior gene elongation factor 1-alpha 1 (EF1, Cobs_01649; Klein et al. 2015) was used as a positive control. In addition, infection was assessed in two adult queens from each of five TW colonies to confirm that this population carries the $w$ Cobs-JP strain. Each PCR reaction contained $5 \mu \mathrm{L}$ Taq polymerase (GoTaq, Promega), $3 \mu \mathrm{L} \mathrm{H}_{2} \mathrm{O}, 0.5 \mu \mathrm{L}$ forward primer $(10 \mu \mathrm{M}), 0.5 \mu \mathrm{L}$ reverse primer $(10 \mu \mathrm{M})$, and $1 \mu \mathrm{L}$ DNA. PCRs were run at $94^{\circ} \mathrm{C}$ for $4 \mathrm{~min}$ followed by 36 cycles at $94^{\circ} \mathrm{C}$ for $30 \mathrm{~s}, 57^{\circ} \mathrm{C}$ for $30 \mathrm{~s}$, and $72^{\circ}$ for $30 \mathrm{~s}$, with a final step of $72^{\circ} \mathrm{C}$ for $10 \mathrm{~min}$. Electrophoresis separation of PCR products was performed on a $1.5 \%$ TAE-Gel with $0.5 \mu \mathrm{L}$ of $0.001 \%$ Gel-Red for $45 \mathrm{~min}$ at $60 \mathrm{~V}$ and $65 \mathrm{~mA}$.

\section{POPULATION-SPECIFIC WOIbachia TITERS}

We compared Wolbachia infection titers of adult workers and queens from Brazilian ( $n=9$ workers, $n=10$ queens) and Japanese ( $n=10$ workers, $n=9$ queens) colonies by amplifying a portion of the cytochrome $c$ oxidase subunit 1 gene (coxA_wCobs-for: 5'-TTGGTCATCCAGAAGTTTACGT$3^{\prime}$, coxA_wCobs-rev: 5'-TGAGCCCAAACCATAAAGCC-3') in qPCR. As a relative standard, EFl was used. Each qPCR reaction contained $5 \mu \mathrm{L}$ SYBR (Peqlab), $2 \mu \mathrm{L} \mathrm{H}_{2} \mathrm{O}, 1 \mu \mathrm{L}$ forward primer $(2 \mu \mathrm{M}), 1 \mu \mathrm{L}$ reverse primer $(2 \mu \mathrm{M})$, and $1 \mu \mathrm{L}$ DNA. Reactions were performed in duplicates and run at $95^{\circ} \mathrm{C}$ for $3 \mathrm{~min}$ followed by 40 cycles at $95^{\circ} \mathrm{C}$ for $5 \mathrm{~s}, 60^{\circ} \mathrm{C}$ for $20 \mathrm{~s}$, and $95^{\circ} \mathrm{C}$ for $10 \mathrm{~s}$ and a final melt curve analysis step in which reactions were heated from 65 to $95^{\circ} \mathrm{C}$ in $5^{\circ} \mathrm{C}$ steps. Single-amplicon production was confirmed with melt curve analyses and relative Wolbachia titers calculated with the $2^{-\triangle C T}$ method (Schmittgen and Livak 2008).

\section{AGE AND MORPH-SPECIFIC Wolbachia TITERS}

We quantified Wolbachia titers in individual queens and workers from Brazilian colonies by determining relative cox $A \_w$ Cobs copy numbers with qPCR across developmental stages and adult age classes. In workers, titers were measured in pupae ( $n=4$ white, i.e., young pupae; $n=5$ dark, i.e., old pupae) and in adults 2 days $(n=5), 14$ days $(n=7)$, and 28 days $(n=6)$ posthatching. In queens, titers were measured in pupae ( $n=5$ white pupae, $n=$ 4 dark pupae), as well as in adults 2 days $(n=7), 14$ days $(n=7)$, 28 days ( $n=10$ virgin queens, $n=7$ mated queens), and 48 days $(n=9)$ posthatching. In addition, we quantified cox $A \_w$ Cobs copy numbers in adult morphs ( $n=15$ queens, $n=12$ workers, $n=6$ winged males, $n=7$ wingless males). For all reactions, EF1 was used as a housekeeper. Each qPCR reaction contained 5 $\mu \mathrm{L}$ SYBR (Peqlab), $2 \mu \mathrm{L} \mathrm{H}_{2} \mathrm{O}, 1 \mu \mathrm{L}$ forward primer $(2 \mu \mathrm{M}), 1$ $\mu \mathrm{L}$ reverse primer $(2 \mu \mathrm{M})$, and $1 \mu \mathrm{L}$ DNA. Reactions were performed in triplicates and run as described above. Single-amplicon production was confirmed with melt curve analyses and relative quantities of Wolbachia calculated with the $2^{-\triangle C T}$ method.

\section{RIFAMPICIN EFFECTS ON INFECTION TITERS}

We verified the efficacy of rifampicin treatment by measuring Wolbachia and Cand. Westeberhardia titers in dark worker pupae produced by antibiotic-treated ( $n=21$ from nine colonies) and control colonies ( $n=21$ from eight colonies) using qPCR of $c o x A \_w C o b s$ and $n r d B \_w e C o b s$. In addition, we measured the Wolbachia titers of JP males used in mating combinations BR $\times$ $\mathrm{JP}$ and BR $\times \mathrm{JP}^{\text {rif }+}\left(\mathrm{JP}: n=15 ; \mathrm{JP}^{\text {rif }+}: n=12\right)$ with qPCR on a portion of the JP-specific $w s p$ gene ( $\left.w s p \_w C o b s \_J P\right)$. For all reactions, $E F 1$ was used as a housekeeper. Each qPCR reaction contained $5 \mu \mathrm{L}$ SYBR (Peqlab), $2 \mu \mathrm{L} \mathrm{H}_{2} \mathrm{O}, 1 \mu \mathrm{L}$ forward primer $(2 \mu \mathrm{M}), 1 \mu \mathrm{L}$ reverse primer $(2 \mu \mathrm{M})$, and $1 \mu \mathrm{L}$ DNA. Reactions 
were performed in duplicates (males) or triplicates (worker pupae) and run as described above (except with an annealing temperature of $57^{\circ} \mathrm{C}$ for male pupae). Single-amplicon production was confirmed with melt curve analyses and relative quantities of Wolbachia and Cand. Westeberhardia calculated with the $2^{-\Delta C T}$ method.

\section{HISTOLOGICAL SECTIONING AND FLUORESCENCE IN SITU HYBRIDIZATION}

Cardiocondyla obscurior male abdomens were fixed in $4 \%$ formaldehyde in PBS and washed with water followed by a dehydration series in $n$-butanol $(30 \%, 50 \%, 70 \%, 80 \%, 90 \%, 96 \%)$ at room temperature with shaking for $1 \mathrm{~h}$ at each step. Absolute $n$-butanol was used for the last $3 \mathrm{~h}$ at $30^{\circ} \mathrm{C}$, exchanging the solution each hour. Dehydrated samples were embedded in Technovit 8100 cold polymerizing resin (Heraeus Kulzer, Germany) according to the manufacturer's instructions, cut into semithin sections $(8 \mu \mathrm{m})$ with a Microm HM355S microtome (Thermo Fisher Scientific, Germany), and mounted on glass slides coated with poly-L-lysine (Kindler, Germany). The tissue sections were incubated for $90 \mathrm{~min}$ at $50^{\circ} \mathrm{C}$ in hybridization buffer $(0.9$ $\mathrm{M} \mathrm{NaCl}, 20 \mathrm{mM}$ Tris/HCl [pH 8.0], $0.01 \%$ SDS) containing $0.5 \mu \mathrm{M}$ of the Wolbachia-specific probes Wolb_W2_Cy5 (5'CTTCTGTGAGTACCGTCATTATC-3') (Heddi et al. 1999) and Wolb_Wol3_Cy5 (5'-TCCTCTATCCTCTTTCAATC-3'), (Sanguin et al. 2006), as well as the Cand. Westeberhardia-specific probe Wcard1_Cy3 (5'-ATCAGTTTCGAACGCCATTC-3') (Klein et al. 2015). DAPI (4',6-diamidino-2-phenylindole) was used for host DNA counterstaining. After hybridization, samples were washed with buffer $(0.1 \mathrm{M} \mathrm{NaCl}, 20 \mathrm{mM}$ Tris/ $\mathrm{HCl}$ [pH 8.0], $5 \mathrm{mM}$ EDTA, $0.01 \% \mathrm{SDS}$ ) for $20 \mathrm{~min}$ at $50^{\circ} \mathrm{C}$. Subsequently, the washing buffer was removed and washed with distilled water for $20 \mathrm{~min}$ at $50^{\circ} \mathrm{C}$. Samples were air-dried and covered with VectaShield $^{\circledR}$ (Vector Laboratories, Burlingame, CA, USA) and stored overnight at room temperature. Images were acquired on an AxioImager.Z1 epifluorescence microscope (Carl Zeiss, Jena, Germany).

\section{AUTHOR CONTRIBUTIONS}

CÜ performed the crosses and qPCR. ES analyzed and visualized the data. AMM assembled Wolbachia genomes, performed comparative genomic analyses, calculated the phylogeny, and annotated CI genes. LVF conducted FISH analyses. BS revised the taxonomic status of the populations. JH acquired funding and reviewed and edited the manuscript. JO conceived and supervised the study. JO, ES, and CÜ wrote the original draft of the manuscript. All authors revised and approved the final version of the manuscript.

\section{ACKNOWLEDGMENTS}

The authors thank A. Klein for help with experimental crossings and for analyses of infection titres across morphs and over time. The authors thank J. Wang for help collecting colonies in Taiwan and for providing the $w s p$ sequence of the TW population. The authors thank J. Hacker for help with the BR $\times$ TW crossings and H. Lowack, S. Lorenz and J. Wallner for help with lab work. J. D. Shropshire helped with the cif annotation and B. Weiss assisted with histological preparations. This work was supported by the Marie-Curie AgreenSkills + fellowship program co-funded by the EU's Seventh Framework Program (FP7-609398) to AMM, and a DFG grant He1623/31 to JH and JO.

\section{DATA ARCHIVING}

All genomic data files are available at the Zenodo repository: https:// doi.org/10.5281/zenodo.3561160. All other data have been deposited in Dryad: https://doi.org/10.5061/dryad.dr7sqv9z2.

\section{LITERATURE CITED}

Ahmed, M. Z., J. W. Breinholt, and A. Y. Kawahara. 2016. Evidence for common horizontal transmission of Wolbachia among butterflies and moths. BMC Evol. Biol. 16:118.

Andersen, S. B., M. Boyle, D. R. Nash, and J. J. Boomsma. 2012. Dynamic Wolbachia prevalence in Acromyrmex leaf-cutting ants: potential for a nutritional symbiosis. J. Evol. Biol. 25:1340-1350.

Bankevich, A., S. Nurk, D. Antipov, A. A. Gurevich, M. Dvorkin, A. S. Kulikov, V. M. Lesin, S. I. Nikolenko, S. Pham, A. D. Prjibelski, et al. 2012. SPAdes: a new genome assembly algorithm and its applications to single-cell sequencing. J. Comput. Biol. 19:455-477.

Beckmann, J. F., J. A. Ronau, and M. Hochstrasser. 2017. A Wolbachia deubiquitylating enzyme induces cytoplasmic incompatibility. Nat. Microbiol. 2:17007.

Beckmann, J. F., M. Bonneau, H. Chen, M. Hochstrasser, D. Poinsot, H. Merçot, M. Weill, M. Sicard, and S. Charlat. 2019a. Caution does not preclude predictive and testable models of cytoplasmic incompatibility: a reply to Shropshire et al. Trends Genet. 35:399-400.

Beckmann, J. F., M. Bonneau, H. Chen, M. Hochstrasser, D. Poinsot, H. Merçot, M. Weill, M. Sicard, and S. Charlat. 2019b. The toxin-antidote model of cytoplasmic incompatibility: genetics and evolutionary implications. Trends Genet. 35:175-185.

Bing, X.-L., D.-S. Zhao, J.-T. Sun, K.-J. Zhang, and X.-Y. Hong. 2020. Genomic analysis of Wolbachia from Laodelphax striatellus (Delphacidae, Hemiptera) reveals insights into its "Jekyll and Hyde" mode of infection pattern. Genome Biol. Evol. 12:3818-3831.

Bonneau, M., C. Atyame, M. Beji, F. Justy, M. Cohen-Gonsaud, M. Sicard, and M. Weill. 2018. Culex pipiens crossing type diversity is governed by an amplified and polymorphic operon of Wolbachia. Nat. Commun. 9:319.

Bordenstein, S. R., and J. H. Werren. 2007. Bidirectional incompatibility among divergent Wolbachia and incompatibility level differences among closely related Wolbachia in Nasonia. Heredity 99:278-287.

Braig, H. R., W. Zhou, S. L. Dobson, and S. L. O'Neill. 1998. Cloning and characterization of a gene encoding the major surface protein of the bacterial endosymbiont Wolbachia pipientis. J. Bacteriol. 180:2373-2378.

Branca, A., F. Vavre, J.-F. Silvain, and S. Dupas. 2009. Maintenance of adaptive differentiation by Wolbachia induced bidirectional cytoplasmic incompatibility: the importance of sib-mating and genetic systems. BMC Evol. Biol. 9:185.

Breeuwer, J. A., and J. H. Werren. 1993. Cytoplasmic incompatibility and bacterial density in Nasonia vitripennis. Genetics 135:565-574.

Brucker, R. M., and S. R. Bordenstein. 2012. Speciation by symbiosis. Trends Ecol. Evol. 27:443-451.

. 2013. The hologenomic basis of speciation: gut bacteria cause hybrid lethality in the genus Nasonia. Science 341:667-669. 
Campbell, E. A., N. Korzheva, A. Mustaev, K. Murakami, S. Nair, A. Goldfarb, and S. A. Darst. 2001. Structural mechanism for rifampicin inhibition of bacterial RNA polymerase. Cell 104:901-912.

Chen, F., A. J. Mackey, J. K. Vermunt, and D. S. Roos. 2007. Assessing performance of orthology detection strategies applied to eukaryotic genomes. PLoS ONE 2:e383.

Chen, H., J. A. Ronau, J. F. Beckmann, and M. Hochstrasser. 2019. A Wolbachia nuclease and its binding partner provide a distinct mechanism for cytoplasmic incompatibility. Proc. Natl. Acad. Sci. USA 116:2231422321.

Cheng, D., S. Chen, Y. Huang, N. E. Pierce, M. Riegler, F. Yang, L. Zeng, Y. Lu, G. Liang, and Y. Xu. 2019. Symbiotic microbiota may reflect host adaptation by resident to invasive ant species. PLoS Pathog. 15:e1007942.

Clark, M. E., C. Bailey-Jourdain, P. M. Ferree, S. J. England, W. Sullivan, D. M. Windsor, and J. H. Werren. 2008. Wolbachia modification of sperm does not always require residence within developing sperm. Heredity 101:420-428.

Cooper, B. S., P. S. Ginsberg, M. Turelli, and D. R. Matute. 2017. Wolbachia in the Drosophila yakuba complex: pervasive frequency variation and weak cytoplasmic incompatibility, but no apparent effect on reproductive isolation. Genetics 205:333-351.

Egas, M., F. Vala, and J. A. J. H. Breeuwer. 2002. On the evolution of cytoplasmic incompatibility in haplodiploid species. Evolution 56:11011109.

Engelstädter, J., and A. Telschow. 2009. Cytoplasmic incompatibility and host population structure. Heredity 103:196-207.

Foucaud, J., J. Orivel, A. Loiseau, J. H. C. Delabie, H. Jourdan, D. Konghouleux, M. Vonshak, M. Tindo, J.-L. Mercier, D. Fresneau, et al. 2010. Worldwide invasion by the little fire ant: routes of introduction and eco-evolutionary pathways. Evol. Appl. 3:363-374.

Fry, A. J., M. R. Palmer, and D. M. Rand. 2004. Variable fitness effects of Wolbachia infection in Drosophila melanogaster. Heredity 93:379389.

Goryacheva, I., A. Blekhman, B. Andrianov, and I. Zakharov. 2016. Heritable bacterial endosymbionts in native and invasive populations of Harmonia axyridis. Biol. Invasions 19:493-502.

Gotzek, D., H. J. Axen, A. V. Suarez, S. Helms Cahan, and D. Shoemaker. 2015. Global invasion history of the tropical fire ant: a stowaway on the first global trade routes. Mol. Ecol. 24:374-388.

Heddi, A., A. M. Grenier, C. Khatchadourian, H. Charles, and P. Nardon. 1999. Four intracellular genomes direct weevil biology: nuclear, mitochondrial, principal endosymbiont, and Wolbachia. Proc. Natl. Acad. Sci. 96:6814-6819.

Heinze, J. 2017. Life-history evolution in ants: the case of Cardiocondyla. Proc. Biol. Sci. 284:20161406.

Heinze, J., and B. Hölldobler. 1993. Fighting for a harem of queens: physiology of reproduction in Cardiocondyla male ants. Proc. Natl. Acad. Sci. 90:8412-8414.

Heinze, J., S. Cremer, N. Eckl, and A. Schrempf. 2006. Stealthy invaders: the biology of Cardiocondyla tramp ants. Insect. Soc. 53:1-7.

Hoffmann, A. A., D. Clancy, and J. Duncan. 1996. Naturally-occurring Wolbachia infection in Drosophila simulans that does not cause cytoplasmic incompatibility. Heredity 76:1-8.

Hosokawa, T., R. Koga, Y. Kikuchi, X.-Y. Meng, and T. Fukatsu. 2010. Wolbachia as a bacteriocyte-associated nutritional mutualist. Proc. Natl. Acad. Sci. USA 107:769-774.

Jaenike, J., K. A. Dyer, C. Cornish, and M. S. Minhas. 2006. Asymmetrical reinforcement and Wolbachia infection in Drosophila. PLoS Biol. 4:e325.
Kautz, S., B. E. R. Rubin, and C. S. Moreau. 2013. Bacterial infections across the ants: frequency and prevalence of Wolbachia, Spiroplasma, and Asaia. Psyche 2013:936341.

Kelly, M., S. L. Price, M. de Oliveira Ramalho, and C. S. Moreau. 2019. Diversity of Wolbachia associated with the giant turtle ant, Cephalotes atratus. Curr. Microbiol. 76:1330-1337.

Klein, A. 2015. Endosymbiosis, co-option and recombination: mechanisms to generate evolutionary novelty in the ant Cardiocondyla obscurior. Doctoral diss., Universität Regensburg, Regensburg, Germany.

Klein, A., L. Schrader, R. Gil, A. Manzano-Marín, L. Flórez, D. Wheeler, J. H. Werren, A. Latorre, J. Heinze, M. Kaltenpoth, et al. 2015. A novel intracellular mutualistic bacterium in the invasive ant Cardiocondyla obscurior. ISME J. 10:376-388.

Kurtz, S., A. Phillippy, A. L. Delcher, M. Smoot, M. Shumway, C. Antonescu, and S. L. Salzberg. 2004. Versatile and open software for comparing large genomes. Genome Biol. 5:R12.

Langmead, B., and S. L. Salzberg. 2012. Fast gapped-read alignment with Bowtie 2. Nat. Methods 9:357-359.

Lee, C.-C., C.-Y. Lin, S.-P. Tseng, K. Matsuura, and C.-C. S. Yang. 2020. Ongoing coevolution of Wolbachia and a widespread invasive ant, Anoplolepis gracilipes. Microorganisms 8:1569.

LePage, D. P., J. A. Metcalf, S. R. Bordenstein, J. On, J. I. Perlmutter, J. D. Shropshire, E. M. Layton, L. J. Funkhouser-Jones, J. F. Beckmann, and S. R. Bordenstein. 2017. Prophage WO genes recapitulate and enhance Wolbachia-induced cytoplasmic incompatibility. Nature 543:243-247.

Li, L. 2003. OrthoMCL: identification of ortholog groups for eukaryotic genomes. Genome Res. 13:2178-2189.

Lindsey, A. R. I., D. W. Rice, S. R. Bordenstein, A. W. Brooks, S. R. Bordenstein, and I. L. G. Newton. 2018. Evolutionary genetics of cytoplasmic incompatibility genes cifA and cifB in prophage WO of Wolbachia. Genome Biol. Evol. 10:434-451.

Lis, A., A. Maryańska-Nadachowska, and Ł. Kajtoch. 2015. Relations of Wolbachia infection with phylogeography of Philaenus spumarius (Hemiptera: Aphrophoridae) populations within and beyond the Carpathian contact zone. Microb. Ecol. 70:509-521.

Martinez, J., L. Klasson, J. J. Welch, and F. M. Jiggins. 2021. Life and death of selfish genes: comparative genomics reveals the dynamic evolution of cytoplasmic incompatibility. Mol. Biol. Evol. 38:2-15.

Moreira, L. A., I. Iturbe-Ormaetxe, J. A. Jeffery, G. Lu, A. T. Pyke, L. M. Hedges, B. C. Rocha, S. Hall-Mendelin, A. Day, M. Riegler, et al. 2009. A Wolbachia symbiont in Aedes aegypti limits infection with dengue, Chikungunya, and Plasmodium. Cell 139:1268-1278.

Morrow, J. L., A. A. G. Hall, and M. Riegler. 2017. Symbionts in waiting: the dynamics of incipient endosymbiont complementation and replacement in minimal bacterial communities of psyllids. Microbiome 5:58.

Parker, E. S., I. L. G. Newton, and A. P. Moczek. 2020. (My microbiome) would walk 10,000 miles: maintenance and turnover of microbial communities in introduced dung beetles. Microb. Ecol. 80:435-446.

Perlmutter, J. I., S. R. Bordenstein, R. L. Unckless, D. P. LePage, J. A. Metcalf, T. Hill, J. Martinez, F. M. Jiggins, and S. R. Bordenstein. 2019. The phage gene $w m k$ is a candidate for male killing by a bacterial endosymbiont. PLoS Pathog. 15:e1007936.

Perrot-Minnot, M. J., and J. H. Werren. 1999. Wolbachia infection and incompatibility dynamics in experimental selection lines. J. Evol. Biol. 12:272-282.

Perrot-Minnot, M. J., B. Cheval, A. Migeon, and M. Navajas. 2002. Contrasting effects of Wolbachia on cytoplasmic incompatibility and fecundity in the haplodiploid mite Tetranychus urticae. J. Evol. Biol. 15:808-817.

Pontieri, L., A. M. Schmidt, R. Singh, J. S. Pedersen, and T. A. Linksvayer. 2017. Artificial selection on ant female caste ratio uncovers a link be- 
tween female-biased sex ratios and infection by Wolbachia endosymbionts. J. Evol. Biol. 30:225-234.

Ronquist, F., M. Teslenko, P. van der Mark, D. L. Ayres, A. Darling, S. Höhna, B. Larget, L. Liu, M. A. Suchard, and J. P. Huelsenbeck. 2012. MrBayes 3.2: efficient Bayesian phylogenetic inference and model choice across a large model space. Syst. Biol. 61:539-542.

Russell, J. A. 2012. The ants (Hymenoptera: Formicidae) are unique and enigmatic hosts of prevalent Wolbachia (Alphaproteobacteria) symbionts. Myrmecol. News 16:7-23.

Russell, J. A., B. Goldman-Huertas, C. S. Moreau, L. Baldo, J. K. Stahlhut, J. H. Werren, and N. E. Pierce. 2009. Specialization and geographic isolation among Wolbachia symbionts from ants and lycaenid butterflies. Evolution 63:624-640.

Sanguin, H., A. Herrera, C. Oger-Desfeux, A. Dechesne, P. Simonet, E. Navarro, T. M. Vogel, Y. Moënne-Loccoz, X. Nesme, and G. L. Grundmann. 2006. Development and validation of a prototype $16 \mathrm{~S}$ rRNA-based taxonomic microarray for Alphaproteobacteria. Environ. Microbiol. 8:289-307.

Schmidt, C. V., S. Frohschammer, A. Schrempf, and J. Heinze. 2013. Virgin ant queens mate with their own sons to avoid failure at colony foundation. Naturwissenschaften 101:69-72.

Schmittgen, T. D., and K. J. Livak. 2008. Analyzing real-time PCR data by the comparative CT method. Nat. Protoc. 3:1101-1108.

Schrader, L., J. W. Kim, D. Ence, A. Zimin, A. Klein, K. Wyschetzki, T. Weichselgartner, C. Kemena, J. Stökl, E. Schultner, et al. 2014. Transposable element islands facilitate adaptation to novel environments in an invasive species. Nat. Commun. 5:5495.

Schrader, L., D. F. Simola, J. Heinze, and J. Oettler. 2015. Sphingolipids, transcription factors, and conserved toolkit genes: developmental plasticity in the ant Cardiocondyla obscurior. Mol. Biol. Evol. 32:14741486.

Schrempf, A., and J. Heinze. 2006. Proximate mechanisms of male morph determination in the ant Cardiocondyla obscurior. Evol. Dev. 8:266272.

Schrempf, A., E. Darrouzet, and J. Heinze. 2007. Mating success and potential male-worker conflict in a male-dimorphic ant. BMC Evol. Biol. 7:114.

Schrempf, A., K. von Wyschetzki, A. Klein, L. Schrader, J. Oettler, and J. Heinze. 2015. Mating with an allopatric male triggers immune response and decreases longevity of ant queens. Mol. Ecol. 24:3618-3627.

Schrempf, A., A. Moser, J. Delabie, and J. Heinze. 2016. Sperm traits differ between winged and wingless males of the ant Cardiocondyla obscurior. Integr. Zool. 11:427-432.

Schuler, H., C. Bertheau, S. P. Egan, J. L. Feder, M. Riegler, B. C. SchlickSteiner, F. M. Steiner, J. Johannesen, P. Kern, K. Tuba, et al. 2013. Evidence for a recent horizontal transmission and spatial spread of Wolbachia from endemic Rhagoletis cerasi (Diptera: Tephritidae) to invasive Rhagoletis cingulata in Europe. Mol. Ecol. 22:4101-4111.

Schultner, E., and U. Pulliainen. 2020. Brood recognition and discrimination in ants. Insect. Soc. 67:11-34.

Seemann, T. 2014. Prokka: rapid prokaryotic genome annotation. Bioinformatics 30:2068-2069.

Seifert, B., M. Ritz, and S. Csôsz. 2013. Application of exploratory data analyses opens a new perspective in morphology-based alpha-taxonomy of eusocial organisms. Myrmecol. News 19:1-15.

Seifert, B., I. Okita, and J. Heinze. 2017. A taxonomic revision of the Cardiocondyla nuda group (Hymenoptera: Formicidae). Zootaxa 4290:324356.

Shoemaker, D. D., V. Katju, and J. Jaenike. 1999. Wolbachia and the evolution of reproductive isolation between Drosophila recens and Drosophila subquinaria. Evolution 53:1157-1164.
Shropshire, J. D., and S. R. Bordenstein. 2019. Two-by-one model of cytoplasmic incompatibility: synthetic recapitulation by transgenic expression of cifA and cifB in Drosophila. PLoS Genet. 15:e1008221.

Shropshire, J. D., J. On, E. M. Layton, H. Zhou, and S. R. Bordenstein. 2018. One prophage WO gene rescues cytoplasmic incompatibility in Drosophila melanogaster. Proc. Natl. Acad. Sci. USA 115:49874991.

Shropshire, J. D., B. Leigh, S. R. Bordenstein, A. Duplouy, M. Riegler, J. C. Brownlie, and S. R. Bordenstein. 2019. Models and nomenclature for cytoplasmic incompatibility: caution over premature conclusions - a response to Beckmann et al. Trends Genet. 35:397-399.

Shropshire, J. D., B. Leigh, and S. R. Bordenstein. 2020. Symbiont-mediated cytoplasmic incompatibility: what have we learned in 50 years? eLife 9:e61989.

Telschow, A., N. Yamamura, and J. H. Werren. 2005. Bidirectional cytoplasmic incompatibility and the stable coexistence of two Wolbachia strains in parapatric host populations. J. Theor. Biol. 235:265-274.

Telschow, A., M. Flor, Y. Kobayashi, P. Hammerstein, and J. H. Werren. 2007. Wolbachia-induced unidirectional cytoplasmic incompatibility and speciation: mainland-island model. PLoS ONE 2:e701.

Tolley, S. J. A., P. Nonacs, and P. Sapountzis. 2019. Wolbachia horizontal transmission events in ants: what do we know and what can we learn? Front. Microbiol. 10:296.

Treanor, D., T. Pamminger, and W. O. H. Hughes. 2018. The evolution of caste-biasing symbionts in the social hymenoptera. Insect. Soc. 65:513519.

Tseng, S.-P., J. K. Wetterer, A. V. Suarez, C.-Y. Lee, T. Yoshimura, D. Shoemaker, and C.-C. S. Yang. 2019. Genetic diversity and Wolbachia infection patterns in a globally distributed invasive ant. Front. Genet. 10:838.

Tsutsui, N. D., A. V. Suarez, D. A. Holway, and T. J. Case. 2000. Reduced genetic variation and the success of an invasive species. Proc. Natl. Acad. Sci. USA 97:5948-5953.

Vala, F., J. A. Breeuwer, and M. W. Sabelis. 2000. Wolbachia-induced "hybrid breakdown" in the two-spotted spider mite Tetranychus urticae Koch. Proc. R. Soc. Lond. B Biol. Sci. 267:1931-1937.

Vavre, F., F. Fleury, J. Varaldi, P. Fouillet, and M. Bouletreau. 2000. Evidence for female mortality in Wolbachia-mediated cytoplasmic incompatibility in haplodiploid insects: epidemiologic and evolutionary consequences. Evolution 54:191-200.

Veneti, Z., M. E. Clark, S. Zabalou, T. L. Karr, C. Savakis, and K. Bourtzis. 2003. Cytoplasmic incompatibility and sperm cyst infection in different Drosophila-Wolbachia associations. Genetics 164:545552.

Versace, E., V. Nolte, R. V. Pandey, R. Tobler, and C. Schlötterer. 2014. Experimental evolution reveals habitat-specific fitness dynamics among Wolbachia clades in Drosophila melanogaster. Mol. Ecol. 23:802814.

Wenseleers, T., L. Sundström, and J. Billen. 2002. Deleterious Wolbachia in the ant Formica truncorum. Proc. R. Soc. Lond. B Biol. Sci. 269:623629.

Werren, J. H., L. Baldo, and M. E. Clark. 2008. Wolbachia: master manipulators of invertebrate biology. Nat. Rev. Microbiol. 6:741-751.

Wick, R. R., M. B. Schultz, J. Zobel, and K. E. Holt. 2015. Bandage: interactive visualization of de novo genome assemblies. Bioinformatics 31:3350-3352.

Yen, J. H., and A. R. Barr. 1971. New hypothesis of the cause of cytoplasmic incompatibility in Culex pipiens L. Nature 232:657-658.

Zepeda-Paulo, F., S. Ortiz-Martínez, A. X. Silva, and B. Lavandero. 2018. Low bacterial community diversity in two introduced aphid pests revealed with $16 \mathrm{~S}$ rRNA amplicon sequencing. PeerJ 6:e4725. 
Zimmermann, L., A. Stephens, S.-Z. Nam, D. Rau, J. Kübler, M. Lozajic, F. Gabler, J. Söding, A. N. Lupas, and V. Alva. 2018. A completely reimplemented MPI bioinformatics toolkit with a new HHpred server at its core. J. Mol. Biol. 430:2237-2243.
Zug, R., and P. Hammerstein. 2012. Still a host of hosts for Wolbachia: analysis of recent data suggests that $40 \%$ of terrestrial arthropod species are infected. PLoS ONE 7:e38544.

\section{Associate Editor: T. Giraud Handling Editor: A. G. McAdam}

\section{Supporting Information}

Additional supporting information may be found online in the Supporting Information section at the end of the article.

Figure S1: Cytoplasmic incompatibility between populations consistently reduces colony fitness.

Figure S2: Maximum weekly egg numbers produced by interpopulation crosses Maximum weekly egg numbers produced by Brazilian (BR) and Japanese (JP) queens mated to males from their own or from a different population.

Figure S3: Total number of male pupae produced in interpopulation crosses Total number of male pupae produced in crosses between Brazilian (BR) and Japanese (JP) males and queens monitored for 12 weeks.

Figure S4: Effects of rifampicin treatment on endosymbiont titers in worker pupae Rifampicin treatment significantly decreases Cand.

Figure S5: Effects of tetracycline treatment on Wolbachia titers in workers Tetracycline treatment (0.5\% in a 1:1 honey-water solution) increases Wolbachia titers in worker pupae.

Figure S6: Recovery of endosymbiont titers after rifampicin treatment Recovery of Cand. Westeberhardia cardiocondylae (A) and Wolbachia (B) titers in workers 6 months after rifampicin treatment.

Figure S7: Wolbachia surface protein sequences differ between populations Alignment of Wolbachia surface protein (wsp) primers and sequences obtained from C. obscurior samples collected in Brazil (BR), Japan (JP), and Taiwan (TW).

Figure S8: Unidirectional cytoplasmic incompatibility between populations Mean (A) and maximum (B) weekly egg numbers produced by crosses between queens and males from Taiwan (TW), Japan (JP), and Brazil (BR).

Figure S9: Population-specific Wolbachia titers.

Figure S10: Morph-specific Wolbachia titers.

Figure S11: Age-dependent changes in Wolbachia titers in queens and workers.

Table S1: Bonferroni-Holm corrected $P$-values for pairwise comparisons of mean egg numbers produced by crosses between queens and males from Brazil (BR) and Japan (JP) during the first 6 weeks.

Table S2: Bonferroni-Holm corrected $P$-values for pairwise comparisons of maximum egg numbers produced by crosses between queens and males from Brazil (BR) and Japan (JP) during the first 6 weeks.

Table S3: Bonferroni-Holm corrected $P$-values for pairwise comparisons of inferred hatching rates of eggs produced by crosses between queens and males from Brazil (BR) and Japan (JP).

Table S4: Bonferroni-Holm corrected $P$-values for pairwise comparisons of total pupae numbers produced by crosses between queens and males from Brazil (BR) and Japan (JP) during 12 weeks.

Table S5: Bonferroni-Holm corrected $P$-values for pairwise comparisons of sex ratios produced by crosses between queens and males from Brazil (BR) and Japan (JP) during 12 weeks.

Table S6: Bonferroni-Holm corrected $P$-values for pairwise comparisons of mean egg numbers produced by crosses between queens and males from Brazil (BR), Japan (JP), and Taiwan (TW) during the first 6 weeks.

Table S7: Bonferroni-Holm corrected $P$-values for pairwise comparisons of maximum egg numbers produced by crosses between queens and males from Brazil (BR), Japan (JP), and Taiwan (TW) during the first 6 weeks.

Table S8: Organisms and accession numbers used for BLASTX binning of assembled scaffolds.

Table S9: Organism details of Wolbachia strains and outgroup bacteria used for Bayesian phylogenetic placement of newly sequenced Wolbachia strains. 